\title{
ON AUSLANDER-REITEN COMPONENTS OF BLOCKS AND SELF-INJECTIVE BISERIAL ALGEBRAS
}

\author{
KARIN ERDMANN AND ANDRZEJ SKOWROŃSKI
}

\begin{abstract}
We investigate the existence of Auslander-Reiten components of Euclidean type for special biserial self-injective algebras and for blocks of group algebras. In particular we obtain a complete description of stable AuslanderReiten quivers for the tame self-injective algebras considered here.
\end{abstract}

\section{INTRODUCTION}

Throughout the paper $K$ denotes a fixed algebraically closed field. We shall use the term algebra to mean finite dimensional $K$-algebra and the term module to mean finite dimensional left module.

Special biserial algebras form an important class of tame algebras. Wellknown examples of such algebras are blocks of group algebras with cyclic or dihedral defect groups and algebras appearing in the Gelfand-Ponomarev classification of Harish-Chandra modules over the Lorentz group [GP].

Let $\Lambda$ be a special biserial algebra of infinite type. It is known that any nonperiodic component of the stable Auslander-Reiten quiver of $\Lambda$ is isomorphic to $\mathbf{Z} A_{\infty}^{\infty} / G$ where $G$ is some group of automorphisms. One would like to know when nontrivial groups occur, that is, when the component is Euclidean.

In this paper, we shall prove that for special biserial self-injective algebras, this is closely related to the growth type of the algebra: $\Lambda$ has a Euclidean component if and only if $\Lambda$ is domestic. If so then it has a finite number of Euclidean components, all of them isomorphic to $\mathbf{Z} \widetilde{A_{p}, q}$ where $\Lambda$ is of tubular type $(p, q)$. Otherwise, there are infinitely many components $\cong \mathbf{Z} A_{\infty}^{\infty}$. Detailed statements of the results are given in $\S 2$. The proofs use covering techniques and exploit results by [BR, DS, PS] on biserial and special biserial algebras.

The work was partly motivated by block theory: A number of special biserial symmetric algebras appear in the context of classifying dihedral blocks [E1, E2]. It was known that some of these have Euclidean components, such as 4-dimensional local symmetric special biserial algebras, and also the group algebras of the alternating groups $A_{4}$ and $A_{5}$ over fields of characteristic 2 .

Our results enable us now to give a complete answer. There are indeed a number of domestic algebras amongst the algebras of dihedral type. The only

Received by the editors November 10, 1989.

1980 Mathematics Subject Classification (1985 Revision). Primary 16A46, 20C20; Secondary 16A64, $20 \mathrm{C} 05$. 
ones which are blocks are those which arise when the defect groups are Klein 4-groups. It is convenient to know that the parameters $p, q$ for a $\mathbf{Z} \widetilde{A}_{p, q^{-}}$ component are also ranks of tubes.

The second problem was to study components of semidihedral blocks. This may be reduced to determine components for the local "semidihedral algebra" (see [E2]). The classification of modules in the form given in [CB2] allows to prove that any regular (see 1.2) nonperiodic component of $\Lambda$ has tree class $A_{\infty}^{\infty}$ or $D_{\infty}$. It is also known that the tree class of the only nonregular component is either $D_{\infty}$ or possibly $\widetilde{D}_{n}$.

After improving our understanding of Okuyama's work [O] we were able to extend his ideas to a more general situation:

We investigate additive functions and give necessary conditions for a selfinjective algebra to have a Euclidean component. We apply this first to local self-injective algebras and settle the second problem. This condition is also used to prove that a group algebra $K G$ has a Euclidean component if and only if the characteristic of $K$ is 2 and $K G$ has a block whose defect groups are Klein 4groups. This generalizes the original theorem of Okuyama; it has been obtained independently by Christine Bessenrodt [Bs].

In the first section we give a summary on biserial algebras, including an outline of the necessary framework on coverings. The second section studies Euclidean components of special biserial self-injective algebras. In the third section we give the necessary condition for existence of Euclidean components and apply it to the local semidihedral algebras and to blocks. The last section contains applications.

For a module $M$, we denote by $\operatorname{soc} M$ and top $M$ the largest semisimple submodule and factor module respectively. We sometimes write $|M|$ for $\operatorname{dim}_{K} M$. Concerning further notation, we refer to [R2, Be, HB].

\section{Preliminaries on biserial algebras}

Throughout this section algebras are assumed to be basic and connected.

1.1 Let $R$ be a locally bounded $K$-category [BG]. An $R$-module is a covariant $K$-linear functor $M$ from $F$ to the category of $K$-vector spaces. We denote by $\bmod R$ the category of all finite dimensional $R$-modules. If $R$ is finite (the number of objects is finite), then $\bmod R$ is equivalent to the category $\bmod A$ of left modules over the associated matrix algebra $\bigoplus R$ consisting of all matrices $\left(a_{y x}\right)_{x, y \in R}$ where $a_{y x} \in R(x, y)$. It is well known that each (finite dimensional) algebra is isomorphic to a matrix algebra $\oplus R$ for some finite category $R$.

For a group $G$ of $K$-linear automorphisms of $R$ acting freely on the objects of $R$, we denote by $R / G$ the quotient category whose objects are the $G$-orbits of the objects of $R$. Then there is a Galois covering functor $F: R \rightarrow R / G$ which assigns to each object $x$ its $G$-orbit $G x$. We denote by $F_{\lambda}: \bmod R \rightarrow$ $\bmod R / G$ the associated push-down functor [BG, 3.2]. A group $G$ of $K$-linear automorphisms of $R$ is called admissible if its action on the objects is free and has finitely many orbits. Then $R / G$ is defined and $\bigoplus(R / G)$ is an algebra. A locally bounded category $R$ is called simply connected [AS] if it is triangular (its ordinary quiver has no oriented cycles) and for any presentation $R \stackrel{\sim}{\rightarrow} k Q / I$ as a bound quiver category, the fundamental group $\Pi_{1}(Q, I)$ of $(Q, I)$ is trivial. 
This is equivalent to the fact that $R$ is triangular and each Galois covering of $R$ is trivial. A locally bounded category $\Lambda$ is called standard [S2] if it admits a Galois covering $R \rightarrow \Lambda$ with $R$ simply connected. Finally, an algebra $A$ is standard if it is isomorphic to $\bigoplus \Lambda$ for some (finite) standard category $\Lambda$.

1.2 For a locally bounded category $R$ we denote by $\Gamma_{R}$ the AuslanderReiten quiver of $R$. We shall use freely properties of Auslander-Reiten sequences and the Auslander-Reiten operators $\tau=D T r$ and $\tau^{-1}=\operatorname{Tr} D$, for which we refer to [AR, R2]. We shall agree to identify the points of $\Gamma_{R}$ with the corresponding indecomposable $R$-modules. An indecomposable module $M$ is called stable (resp. periodic) if $\tau^{n} M ¥ 0$ and $\tau^{-n} M \# 0$ for all $n \in \mathbf{N}$ (resp. $\tau^{n} M \cong M$ for some $n \in \mathbf{N}$ ). The full translation subquiver ${ }_{s} \Gamma_{R}$ of $\Gamma_{R}$ consisting of the isomorphism classes of stable modules is called the stable Auslander-Reiten quiver of $R$. According to Riedtmann [Rd], any stable connected translation quiver $\Gamma$ is of the form $\mathrm{ZT} / G$ where $T$ is an oriented (valued) tree, called the tree class of $\Gamma$, and $G$ is an admissible automorphism group. It has been shown in [HPR] that the tree class of any component of the stable Auslander-Reiten quiver of an algebra containing periodic modules is equal to $A_{\infty}$.

We say that a component $\Gamma$ of $\Gamma_{R}$ is regular provided there is no projective and no injective module in $\Gamma$. For the shape of the translation quivers $\mathbf{Z} A_{\infty}^{\infty}$, $\mathbf{Z} A_{\infty}, \mathbf{Z} A_{\infty} /\left\langle\tau^{n}\right\rangle$ we refer to [HPR]. Finally, by $\tilde{A}_{p, q}(p, q \geq 1)$ we mean the quiver

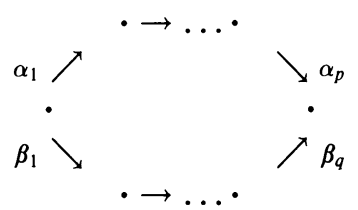

It is well known that, if $\Delta$ is a quiver whose underlying graph is of Euclidean type $\tilde{A}_{n}$, then $\mathbf{Z} \Delta=\mathbf{Z} \widetilde{A}_{p, q}$, where $p$ and $q$ are the numbers of clockwise and counterclockwise oriented arrows in $\Delta$, respectively. Observe that the tree class of $\mathbf{Z} \tilde{A_{p, q}}$ is equal to $A_{\infty}^{\infty}$ for $p q \geq 2$, and $\stackrel{(2,2)}{-} \cdot\left(\tilde{A_{1,2}}\right.$ in the notation of [HPR]) for $p q=1$.

1.3 Let $A$ be an algebra and $K[x]$ be the polynomial algebra in one variable. Following Drozd [D], $A$ is called tame if, for any dimension $d$, there is a finite number of $A-K[x]$-bimodules $Q_{i}, 1 \leq i \leq n_{d}$, which are finitely generated and free as right $K[x]$-modules, and satisfy the following condition:

(a) All but a finite number of isomorphism classes of indecomposable $A$ modules of dimension $d$ are of the form $Q_{i} \otimes_{K[x]} K[x] /(x-\lambda)$ for some $\lambda \in K$ and some $i, 1 \leq i \leq n_{d}$.

Let $\mu_{A}(d)$ be the least number of bimodules $Q_{i}$ satisfying the above condition. Then $A$ is called of polynomial growth [S1] if there is a natural number $m$ such that $\mu_{A}(d) \leq d^{m}$ for all $d \geq 1$.

Further, $A$ is domestic [R1] if there is a finite number of $A-K[x]$-bimodules $Q_{i}, 1 \leq i \leq n$, which are finitely generated free right $K[x]$-modules and satisfy the following condition:

(b) For each dimension $d$, all but a finite number of isomorphism classes of indecomposable $A$-modules of dimension $d$ are of the form $Q_{i} \otimes_{K[x]} V$ for some $i$ and some indecomposable $K[x]$-module $V$. 
Moreover, $A$ is called $n$-parametric if the minimal number of such bimodules is $n$. Every domestic algebra is of polynomial growth (cf. [S3]).

1.4 The repetitive category (cf. [HW]) of a locally bounded category $R$ is the self-injective locally bounded category $\widehat{R}$ whose objects are the pairs $(n, x)=x_{n}, x \in R, n \in \mathbf{Z}$, and $\widehat{R}\left(x_{n}, y_{n}\right)=\{n\} \times R(x, y), \widehat{R}\left(x_{n+1}, y_{n}\right)=$ $\{n\} \times D R(y, x)$, and $\widehat{R}\left(x_{p}, y_{q}\right)=0$ if $p \neq q, q+1$, where $D V$ denotes the dual space $\operatorname{Hom}_{K}(V, K)$ of $V$.

We denote by $\nu_{R}$ the Nakayama automorphism of $\widehat{R}$ which assigns to each object $x_{n}=(n, x)$ the object $x_{n+1}=(n+1, x)$. A $\nu$-slice of $\widehat{R}$ is a full subcategory of $\widehat{R}$ which is connected and does not contain two objects from the same $\nu$-orbit.

1.5 A locally bounded category $R$ is called biserial if the radial of each nonuniserial indecomposable projective left or right $R$-module is a sum of two uniserial submodules whose intersection is simple or zero. A locally bounded category $R$ is special biserial (cf. [SW]) if it is isomorphic to a bound quiver category $K Q / I$, where the bound quiver $(Q, I)$ satisfies the following conditions:

(R1) The number of arrows in $Q$ with a prescribed source or target is at most two,

(R2) For any arrow $\alpha$ of $Q$, there is at most one arrow $\beta$ and one arrow $\gamma$ such that $\alpha \beta$ and $\gamma \alpha$ are not in $I$.

An algebra $A$ is (special) biserial if $A \cong \bigoplus R$ for some (special) biserial category $R$. By [SW] special biserial algebras are biserial, and representationfinite biserial algebras are special biserial. Examples of representation-infinite special biserial algebras are representation-infinite, tilted algebras of type $\widetilde{A}_{p, q}$ [R2, AS1] and blocks with dihedral defect groups in characteristic 2 [E1]. Following [AS1], a triangular locally bounded category $B$ is called gentle if it is isomorphic to $K Q / I$ where the bound quiver $(Q, I)$ satisfies $(\mathrm{R} 1),(\mathrm{R} 2)$ and the following two conditions:

(R3) $I$ is generated by a set of paths of length 2,

(R4) For any arrow $\alpha$ of $Q$, there is at most one arrow $\xi$ and at most one arrow $\eta$ such that $\alpha \xi$ and $\eta \alpha$ belong to $I$.

By a tree category we mean a locally bounded category whose ordinary quiver is a tree. Examples of gentle tree categories are universal Galois coverings of representation-infinite tilted algebras of type $\widetilde{A}_{p, q}$. We shall use the following characterization of self-injective special biserial algebras proved in [PS].

Theorem. Let $A$ be a representation-infinite algebra. Then the following conditions are equivalent:

(i) $A$ is standard, biserial and self-injective.

(ii) $A$ is isomorphic to $\bigoplus(R / G)$ where $R$ is a self-injective simply connected locally bounded $K$-category such that every full finite subcategory is representation-finite, and $G$ is an admissible group of $K$-linear automorphisms of $R$.

(iii) $A$ is isomorphic to $\bigoplus(\widehat{B} / G)$, where $B$ is an infinite locally bounded gentle tree category and $G$ is an admissible torsion-free group of $K$-linear automorphisms of $\widehat{B}$.

(iv) $A$ is self-injective special biserial.

1.6 We shall now describe the indecomposable modules and Auslander- 
Reiten sequences of self-injective special biserial algebra. We use the method based on covering theory [SW, DS]. Let $A$ be a representation-infinite selfinjective special biserial algebra. Then, by $1.5, A \stackrel{\sim}{\rightarrow} \bigoplus(R / G)$ where $R=\widehat{B}$ is the repetitive category of an infinite gentle tree category $B$ and $G$ is an admissible torsionfree group of $K$-linear automorphisms of $\widehat{B}$. Moreover, by [PS, Lemma 8 ], $R=\widehat{B}$ is a special biserial simply connected self-injective category such that every full finite subcategory is representation-finite. A line in $R$ is a full convex subcategory $L$ of $R$ which is isomorphic to the path category of a linear quiver (of type $A_{n}, A_{\infty}$ or $A_{\infty}^{\infty}$ ). A line $L$ is $G$-periodic if its stablizer $G_{L}=\{g \in G: g L=L\}$ is nontrivial. With each line $L$ in $R$ we associate a canonical indecomposable $R$-module $M(L)$ by setting $M(L)(x)=K$ for $x \in L, M(L)(x)=0$ for $x \notin L$ and $M(L)(\gamma)=\mathrm{id}_{K}$ for each path $\gamma$ in $L$. It is well known that the modules $M(L)$ where $L$ ranges over all finite lines in $R$, are representatives of isoclasses of all (finite-dimensional) nonprojective indecomposable $R$-modules. The indecomposable projective $R$-modules are given by the "commutative squares" of $R$. Denote by $F_{\lambda}: \bmod R \rightarrow \bmod R / G$ the push-down functor associated with the Galois covering $F: R \rightarrow R / G$. Following [DS] there are two kinds of indecomposable $R / G$-modules: modules of the first kind formed by all indecomposable modules of the form $F_{\lambda}(M)$, $M \in \bmod R$, and the remaining modules called modules of the second kind. Hence every nonprojective indecomposable $R / G$-module of the first kind is isomorphic to $F_{\lambda} M(L)$ for some finite line $L$ in $R$. The indecomposable $R / G$-modules of the second kind can be described as follows. Let $\mathscr{L}$ be the set of all $G$-periodic lines in $R$ and $\mathscr{L}_{0}$ a fixed set of representatives of the $G$-orbits in $\mathscr{L}$. Then, for any $L \in \mathscr{L}_{0}$, the canonical action of $G_{L}$ on $L$ supplies a right $K G_{L}$-module structure on $F_{\lambda} M(L)$, and for each $a \in R / G$, $F_{\lambda} M(L)(a)$ is a free $K G_{L}$-module of finite rank. Since $G_{L}$ is an infinite cyclic group, $K G_{L} \cong K\left[x, x^{-1}\right]$ and we have a functor

$$
\phi^{L}=F_{\lambda} M(L) \otimes_{K\left[x, x^{-1}\right]}-: \bmod K\left[x, x^{-1}\right] \rightarrow \bmod R / G .
$$

It was shown in [DS, Theorem 3.6] that every indecomposable $R / G$-module of the second kind is isomorphic to $\phi^{L}(V)$ for some $L \in \mathscr{L}_{0}$ and some indecomposable finite dimensional $K\left[x, x^{-1}\right]$-module $V$.

Observe that the family of ordinary quivers of $F(L), L \in \mathscr{L}$, coincides with the family of primitive walks (in the sense of [WW]). The above description of indecomposable $R / G$-modules shows also that $A$ is tame (cf. [WW, DS, BR]).

We shall now describe the Auslander-Reiten sequences in $\bmod R / G$. From [DS, Theorem 3.6] the Auslander-Reiten sequences terminating at the indecomposable $R / G$-modules of the second kind are of the form

$$
0 \rightarrow \phi^{L} N_{\lambda}(i) \rightarrow \phi^{L} N_{\lambda}(i-1) \oplus \phi^{L} N_{\lambda}(i+1) \rightarrow \phi^{L} N_{\lambda}(i) \rightarrow 0
$$

where $N_{\lambda}(i)=K\left[x, x^{-1}\right] /(x-\lambda)^{i}$ with $\lambda \in K^{*}, i \geq 1$ and $N_{\lambda}(0)=0$. In particular, $\Gamma_{A} \cong \Gamma_{R / G}$ is isomorphic to the disjoint union of translation quivers

$$
\left(\Gamma_{R} / G\right) \amalg\left(\coprod_{L \in \mathscr{L}_{0}} \mathscr{T}_{L}\right)
$$

where for each $L \in \mathscr{L}_{0}, \mathscr{T}_{L}$ is a $K^{*}$-family of stable tubes of rank 1 . The Auslander-Reiten sequences whose middle term contains an indecomposable 
projective (injective) direct summand $P$ are of the form

$$
0 \rightarrow \operatorname{rad} P \rightarrow \operatorname{rad} P / \operatorname{soc} P \oplus P \rightarrow P / \operatorname{soc} P \rightarrow 0 .
$$

Assume now that $F_{\lambda} M(L)$ is a nonprojective indecomposable $R / G$-module of the first kind which is not isomorphic to $P / \operatorname{soc} P$ for some projective module $P$. The line $L$ is of the form

$$
e_{j_{0}} \leftarrow \cdots \leftarrow e_{i_{1}} \rightarrow \cdots \rightarrow e_{j_{1}} \leftarrow \cdots \rightarrow e_{j_{t-1}} \leftarrow \cdots e_{k} \leftarrow e_{i_{t}} \rightarrow \cdots \rightarrow e_{j_{t}}
$$

where the first or the last subpath may be trivial. Let $L^{r}$ be the maximal line in $R$ extending $L$ on the right by $e_{j_{t}} \rightarrow e_{j_{t+1}} \leftarrow \cdots \leftarrow e_{i_{t+1}}$ that is,

$$
L^{r}: e_{j_{0}} \leftarrow \cdots \leftarrow e_{j_{1}} \rightarrow \cdots \cdots \rightarrow e_{j_{t}} \rightarrow e_{j_{t+1}} \leftarrow \cdots \leftarrow e_{i_{t+1}}
$$

if such a line exists. If not, let $L^{r}$ be the line obtained from $L$ by cancellation of the last subpath including the vertex $e_{i_{t}}$ (thus $L^{r}$ may be empty), that is,

$$
L^{r}: e_{j_{0}} \leftarrow \cdots \leftarrow e_{j_{1}} \rightarrow \cdots \rightarrow e_{j_{t-1}} \leftarrow \cdots \leftarrow e_{k} .
$$

Similarly let ${ }^{l} L$ be obtained from $L$ by the corresponding operations on the left-hand side of $L$. Since $M(L)$ is nonprojective, at least one of the lines ${ }^{l}\left(L^{r}\right)$ or $\left({ }^{l} L\right)^{r}$ is nonempty, and if both are defined, then ${ }^{l}\left(L^{r}\right)=\left({ }^{l} L\right)^{r}$. We set $\tau L$ to be the nontrivial line ${ }^{l}\left(L^{r}\right)$ or $\left({ }^{l} L\right)^{r}$. Then the Auslander-Reiten sequence terminating at $F_{\lambda} M(L)$ is of the form

$$
0 \rightarrow F_{\lambda} M(\tau L) \rightarrow F_{\lambda} M\left({ }^{l} L\right) \oplus F_{\lambda} M\left(L^{r}\right) \rightarrow F_{\lambda} M(L) \rightarrow 0
$$

(cf. [SW, WW, BR]). Moreover, it has been shown in [BR, §3] that only finitely many such Auslander-Reiten sequences have indecomposable middle terms.

\section{AUSLANDER-REITEN COMPONENTS OF SELF-INJECTIVE SPECIAL BISERIAL ALGEBRAS}

In this section we shall prove the following two theorems on Auslander-Reiten quivers of special biserial self-injective algebras. Throughout this section algebras are assumed to be basic and connected.

2.1 Theorem. Let $A$ be a special biserial self-injective algebra. The following are equivalent:

(i) ${ }_{s} \Gamma_{A}$ has a component of the form $\mathbf{Z} \tilde{A_{p}, q}$.

(ii) ${ }_{s} \Gamma_{A}$ is infinite but has no component of the form $\mathbf{Z} A_{\infty}^{\infty}$.

(iii) There are positive integers $m, p, q$ such that ${ }_{s} \Gamma_{A}$ is a disjoint union of $m$ components of the form $\mathbf{Z} \widetilde{A}_{p, q}, m$ components of the form $\mathbf{Z} A_{\infty} /\left\langle\tau^{p}\right\rangle, m$ components of the form $\mathbf{Z} A_{\infty} /\left\langle\tau^{q}\right\rangle$ and infinitely many components of the form $\mathbf{Z} A_{\infty} /\langle\tau\rangle$.

(iv) All but a finite number of components of $\Gamma_{A}$ are of the form $\mathbf{Z} A_{\infty} /\langle\tau\rangle$.

(v) $A$ is isomorphic to $\bigoplus(\hat{\Lambda} / G)$, where $\Lambda$ is the category associated with a representation-infinite tilted algebra of type $\tilde{A}_{p, q}$ having a complete slice in its preinjective component, and $G$ is an admissible infinite cyclic group of $K$-linear automorphisms of $\hat{\Lambda}$.

(vi) $A \cong K Q / I$ where $(Q, I)$ satisfies (R1), (R2) and the number $e$ of its primitive walks is a positive integer.

(vii) $A$ is representation-infinite domestic.

(viii) $A$ is representation-infinite of polynomial growth. 
2.2 Theorem. Let $A$ be a special biserial self-injective algebra. The following conditions are equivalent:

(i) ${ }_{s} \Gamma_{A}$ has a component of the form $\mathbf{Z} A_{\infty}^{\infty}$.

(ii) $\Gamma_{A}$ has infinitely many (regular) components of the form $\mathbf{Z} A_{\infty}^{\infty}$.

(iii) ${ }_{s} \Gamma_{A}$ is a disjoint union of a finite number of components of the form $\mathbf{Z} A_{\infty} /\left\langle\tau^{n}\right\rangle$ with $n>1$, infinitely many components of the form $\mathbf{Z} A_{\infty} /\langle\tau\rangle$ and infinitely many components of the form $\mathbf{Z} A_{\infty}^{\infty}$.

(iv) $A \cong K Q / I$ where $(Q, I)$ satisfies (R1), (R2) and has infinitely many primitive walks.

(v) $A$ is not of polynomial growth.

In order to prove the theorems we need two propositions.

2.3 Proposition. Let $A$ be a special biserial self-injective algebra and assume that ${ }_{s} \Gamma_{A}$ has a component of the form $\mathbf{Z} \widetilde{A}_{p, q}$. Then $A$ is representation-infinite domestic.

Proof. From Theorem 1.5 we know that $A \cong \bigoplus(\widehat{B} / G)$ where $B$ is an infinite locally bounded gentle tree category and $G$ is an admissible torsion-free group of $K$-linear automorphisms of $\widehat{B}$. Let $R=\widehat{B}, E=R / G$ and $\mathscr{X}$ be a component of $\Gamma_{E}$ whose stable part ${ }_{s} \mathscr{X}$ is of the form $\mathbf{Z} \tilde{A_{p}, q}$. From 1.6 we have

$$
\Gamma_{E}=\left(\Gamma_{R} / G\right) \amalg\left(\coprod_{L \in \mathscr{L}_{0}} \mathscr{T}_{L}\right)
$$

where $\mathscr{T}_{L}, L \in \mathscr{L}_{0}$, are $K^{*}$-families of stable tubes of rank 1 . Therefore there exists a component $\mathscr{C}$ of $\Gamma_{R}$ and an element $1 \neq g \in G$ such that $\mathscr{X} \cong \mathscr{C} /\langle g\rangle$ and ${ }_{s} \mathscr{C} \cong \mathbf{Z} A_{\infty}^{\infty}$. Let the vertices of ${ }_{s} \mathscr{C}$ be given by the indecomposable $R$-modules $M(i, j), i, j \in \mathbf{Z}$, the arrows by $M(i, j-1) \rightarrow M(i, j)$, $M(i-1, j) \rightarrow M(i, j)$, such that $\tau M(i, j) \cong M(i-1, j-1)$ and $g M(i, j) \cong M(i-p, j+q)$. Choose $(i, j) \in \mathbf{Z}^{2}$ such that all projectives in $\mathscr{C}$ are proper successors of modules from the family $\mathscr{S}$ formed by all modules $M(r, s)$ of $\mathscr{C}$ lying on the sectional paths

$$
\begin{aligned}
& M(i+k p, j-k q) \rightarrow \cdots \rightarrow M(i+k p+p, j-k q), \\
& M(i+k p, j-k q) \rightarrow \cdots \rightarrow M(i+k p, j-k q+q), \quad k \in \mathbf{Z} .
\end{aligned}
$$

Without loss of generality we may assume that $i=j=0$.

Denote by $\mathscr{D}$ the full translation subquiver of $\mathscr{C}$ formed by all modules in $\mathscr{C}$ which are predecessors of modules from $\mathscr{S}$ (including the modules from $\mathscr{S}$ ). Since $E$, as a finite special biserial category, is tame, the component $\mathscr{X}$ of $\Gamma_{E}$ contains only finitely many modules of a given dimension. Then, since the push-down functor $F_{\lambda}: \bmod R \rightarrow \bmod (R / G)$ preserves dimension of modules, there are only finitely many $\langle g\rangle$-orbits in $\mathscr{C}$ formed by modules of a given dimension. Therefore, we may also assume that all modules in $\mathscr{D}$ have dimension greater than $2(p+q) \operatorname{dim}_{K} A$. Let $D$ be the full subcategory of $R$ formed by the supports of all indecomposable $R$-modules from $\mathscr{D}$. Observe that $g D=D$. We claim that $D /\langle g\rangle$ is finite. Indeed, consider the quotient category $E^{\prime}=R /\langle g\rangle$ and the push-down functor $F_{\lambda}^{\prime}: \bmod R \rightarrow \bmod E^{\prime}$ associated with the Galois covering $F^{\prime}: R \rightarrow R /\langle g\rangle=E^{\prime}$. Then the (isoclasses of) indecomposable $E^{\prime}$-modules $F_{\lambda}^{\prime} M(i, j), i, j \in \mathbf{Z}$, form all nonprojective 
vertices of a component $\mathscr{X}^{\prime}$ of $\Gamma_{E^{\prime}}$ with ${ }_{s} \chi^{\prime} \cong{ }_{s} \mathscr{C} /\langle g\rangle \cong \mathbf{Z} \tilde{A}_{p, q}$. Let $\mathscr{S}^{\prime}$ be the family of indecomposable $E^{\prime}$-modules of the form $F_{\lambda}^{\prime} M(r, s)$ for all $M(r, s)$ from $\mathscr{S}$. Then all projective modules in $\mathscr{X}^{\prime}$ are proper successors of modules from $\mathscr{S}^{\prime}$. Denote by $D^{\prime}$ the full subcategory of $E^{\prime}$ formed by the supports of the modules in $\mathscr{X}^{\prime}$ which are predecessors of modules from $\mathscr{S}^{\prime}$. Then $D^{\prime} \cong D /\langle g\rangle$. Observe that for any predecessor $F_{\lambda}^{\prime} M(i, j)$ of some module $F_{\lambda}^{\prime} M(r, s) \in \mathscr{S}^{\prime}$ in $\mathscr{X}^{\prime}$ we have an Auslander-Reiten sequence

$$
0 \rightarrow F_{\lambda}^{\prime} M(i-1, j-1) \rightarrow F_{\lambda}^{\prime} M(i, j-1) \oplus F_{\lambda}^{\prime} M(i-1, j) \rightarrow F_{\lambda}^{\prime} M(i, j) \rightarrow 0 \text {. }
$$

Moreover, $F_{\lambda}^{\prime} M(i, j) \cong F_{\lambda}^{\prime} M(i-p, j+q)$ for all $i, j \in \mathbf{Z}$. Hence we deduce that $D^{\prime}$ is the support of the module $\bigoplus_{i=-p}^{0} \bigoplus_{j=-q}^{0} F_{\lambda}^{\prime} M(i, j)$ and consequently $D /\langle g\rangle$ is finite.

We shall now prove that $\bigoplus(D /\langle g\rangle)$ is a representation-infinite tilted algebra of Euclidean type $\widetilde{A}_{p, q}$ having a complete slice in its preinjective component.

First we shall prove that $D$ contains exactly one $G$-periodic line $L$ and that $g L=L$. We know from 1.6 that $M(i, j) \cong M(L(i, j))$ for some finite line $L(i, j)$ in $R$. Moreover, if $M(i, j)$ belongs to $\mathscr{D}$ then it is not a socle factor of an indecomposable projective, and so both lines ${ }^{l} L(i, j)$ and $L(i, j)^{r}$ are nontrivial. Without loss of generality, we may assume that $M(i-1, j) \cong$ $M\left({ }^{l} L(i, j)\right)$ and $M(i, j-1) \cong M\left(L(i, j)^{r}\right)$ and then $M(i-1, j-1) \cong$ $M(\tau L(i, j))=M\left({ }^{l} L(i, j)^{r}\right)$. For a line $W$ in $R$ and a positive integer $m$, we denote by ${ }^{l^{m}} W$ (resp. $\left.W^{r^{m}}\right)$ the line ${ }^{l}\left({ }^{l}\left({ }^{l} W\right) \cdots\right)\left(\right.$ resp. $\left.\left(\cdots\left(W^{r}\right)^{r} \cdots\right)^{r}\right)$ obtained from $W$ by $m$-times applying the left (resp. right) side operation ${ }^{l}()$ (resp. ()$\left.^{r}\right)$. Denote by $L_{0}$ the full subcategory of $R$ formed by the common objects of $L(0,0), L(-p, 0)={ }^{l^{p}} L(0,0)$ and $L(0,-q)=L(0,0)^{r^{q}}$. Observe that $\operatorname{dim}_{K} M\left(L_{0}\right) \geq(p+q) \operatorname{dim}_{K} A$ because the nonzero paths in $R$ have length $<\operatorname{dim}_{K} A$ and $\operatorname{dim}_{K} M(L(0,0))=\operatorname{dim}_{K} M(0,0)>2(p+q) \operatorname{dim}_{K} A$. Hence $L(0,0)$ is of the form

$$
u_{0}-\cdots-x_{0}-\cdots-y_{0}-\cdots-v_{0}
$$

where $L_{0}$ is formed by the line $x_{0}-\cdots-y_{0}$.

Observe that, if $M(i, j) \in \mathscr{D}$ then $M\left[g L(i, j)^{r^{q}}\right] \cong g M(i, j-q) \cong$ $M(i-p, j) \cong M\left[{ }^{p} L(i, j)\right]$. This implies that all differences

$$
a_{m}=\operatorname{dim}_{K} M(-m p-p, 0)-\operatorname{dim}_{K} M(-m p, 0), \quad m \geq 0,
$$

are equal. Similarly, the differences

$$
b_{m}=\operatorname{dim}_{K} M(0,-m q-q)-\operatorname{dim}_{K} M(0,-m q), \quad m \geq 0,
$$

are equal. The modules $M(-m p, 0)$ (resp. $M(0,-m q)), m \geq 0$, belong to pairwise different $\langle g\rangle$-orbits in $\mathscr{C}$. Hence $a_{m}>0$ and $b_{m}>0$. Consequently the lines $L(-m p, 0)$ are of the form

$$
u_{m}-\cdots-x_{m}-\cdots-x_{m-1}-\cdots-x_{1}-\cdots-x_{0}-\cdots-y_{0}-\cdots-v_{0}
$$

and the lines $L(0,-m q)$ of the form

$$
u_{0}-\cdots-x_{0}-\cdots-y_{0}-\cdots-y_{1}-\cdots-y_{m-1}-\cdots-y_{m}-\cdots-v_{m}
$$

where since $g^{m} L(0,-q m)=L(-m p, 0)$, we have $g x_{i}=x_{i+1}, g u_{i}=u_{i+1}$, $g y_{i+1}=y_{i}$ and $g v_{i+1}=v_{i}$ for $i=0, \ldots, m-1$. Moreover, the lines $L(-m p,-m q)$ are of the form

$u_{m}-\cdots-x_{m}-\cdots-x_{m-1}-\cdots-x_{0}-\cdots-y_{0}-\cdots-y_{m-1}-\cdots-y_{m}-\cdots-v_{m}$. 
For each $m \geq 0$, we denote by $L_{m}$ the line

$$
x_{m}-\cdots-x_{0}-\cdots-y_{0}-\cdots-y_{m} \text {. }
$$

Then we have the chain of full subcategories of $R$

$$
L_{0} \subset L_{1} \subset L_{2} \subset \cdots
$$

such that $g L_{m} \subset L_{m+1}$. Consequently $L=\bigcup_{m>0} L_{m}$ is a line in $R$ and $g L=L$. Observe also that, for any $M(i, j)=M(L(i, j))$ from $\mathscr{D}, L(i, j)$ is of the form

$a-\cdots-g^{k} x_{s}-\cdots-g^{k} x_{1}-\cdots-g^{k} x_{0}-\cdots-g^{k} y_{0}-\cdots-g^{k} y_{1}-\cdots-g^{k} y_{t}-\cdots-b$

for some $k \in \mathbf{Z}, s, t \geq 0$, where the line

$$
x_{s}-\cdots-x_{1}-\cdots-x_{0}-\cdots-y_{0}-\cdots-y_{1}-\cdots-y_{t}
$$

is the convex subline of $L_{m}, m=\max (s, t)$. Hence $L$ is the unique $\langle g\rangle$ periodic ( $G$-periodic) line of $D$. Observe also that $L$ is a convex $\nu$-slice in $R=\widehat{B}$ and that $\bigoplus(L /\langle g\rangle)$ is a hereditary algebra of type $\widetilde{A}_{p_{0}}, q_{0}$. We shall show that $\bigoplus(D /\langle g\rangle)$ is a tubular extension of $\bigoplus(L /\langle g\rangle)$ (in the sense of [R2, 4.7]). We claim that for any arrow $\alpha$ in $D$ with source in $L$ its target is also in $L$. Suppose this is not the case. Then there exists $M(i, j)=M(L(i, j))$ in $\mathscr{D}$ such that $g^{k} L(i, j)$ is for some $k \in \mathbf{Z}$, one of the lines

$$
a-\cdots-x_{s}-\cdots-x_{0}-\cdots-y_{0}-\cdots-y_{t}-\cdots \leftarrow c \rightarrow d-\cdots-e
$$

where $c$ lies on $L$ between $y_{t}$ and $y_{t+1}, d \notin L$, or

$$
e-\cdots-d \leftarrow c \rightarrow \cdots-x_{s}-\cdots-x_{0}-\cdots-y_{0}-\cdots-y_{t}-\cdots-b
$$

where $c$ lies on $L$ between $x_{s+1}$ and $x_{s}, d \notin L$. We may assume that $k=0$ and, by symmetry, that $L(i, j)$ is of the first form. Observe that, for any $m \in \mathbf{N}, L(i, j)^{r^{m}}$ either contains $d$ does not contain $c$. On the other hand, $L(i, j)^{r^{q}}=L(i, j-q)$ is of the form

$a-\cdots-x_{s}-\cdots-x_{0}-\cdots-y_{0}-\cdots-y_{t}-\cdots \leftarrow c-\cdots-y_{t+1}-\cdots \leftarrow c^{\prime} \rightarrow d^{\prime}-\cdots-e^{\prime}$

where $c=g c^{\prime}$, a contradiction.

From the choice of $\mathscr{D}$, the support of any module from $\mathscr{D}$ contains a line $g^{k} L_{0}$. Hence the support $L_{i, j}$ of any $M(i, j)$ from $\mathscr{D}$ is of the form

$$
a-\cdots \rightarrow b-\cdots-c \leftarrow \cdots-d
$$

where the common part of $L$ and $L_{i, j}$ is the line between $b$ and $c$, and may be the line $a-\cdots \rightarrow b$ (resp. $c \leftarrow \cdots-d$ ) has only one object. This shows that $D$ is a gentle tree category and a convex $\nu$-slice of $R=\widehat{B}$. Hence, let $\Lambda=D /\langle g\rangle$, then $\bigoplus \Lambda$ is a tubular extension of the hereditary algebra $H=\bigoplus(L /\langle g\rangle)$, and so is a representation-infinite tilted algebra of type $\widetilde{A}_{p^{\prime}}, q^{\prime}, p^{\prime} \geq p_{0}, q^{\prime} \geq q_{0}$, with a complete slice in its preinjective component.

Moreover, $\mathscr{D} /\langle g\rangle$ is a full translation subquiver of a component of $\Gamma_{\Lambda}$. Since any module in $\mathscr{D} /\langle g\rangle$ has infinitely many predecessors and $\mathscr{D} /\langle g\rangle$ is a full translation subquiver of $\mathscr{C} /\langle g\rangle$ whose stable part is of the form $\mathrm{Z} \widetilde{A_{p}, q}$, we infer that $\mathscr{D} /\langle g\rangle$ is a full translation subquiver of the preinjective component of $\Gamma_{\Lambda}$. Consequently $\bigoplus \Lambda$ is a tilted algebra of type $\widetilde{A}_{p, q}$. 
Our next claim is that $\widehat{D}=\widehat{B}$. Suppose that $\widehat{D}$ is a proper subcategory of $\widehat{B}=R$. Since $\widehat{B}$ is connected there exists an object $x \in \widehat{B}, x \notin \widehat{D}$ connected by an arrow to $\widehat{D}$. Then $y=\nu^{r} x$ for some $r \in \mathbf{Z}$ is connected by an arrow to $D$. Obviously $y \notin \widehat{D}$. Let $K$ be the full subcategory of $\widehat{B}$ formed by all objects of $D$ and the objects $g^{m} y, m \in \mathbf{Z}$. Since $K$ is a convex $\nu$-slice in the repetitive category $\widehat{B}$ of the gentle tree category $B$, it is also a gentle tree category. We may assume that $\bigoplus(K /\langle g\rangle)$ is a tubular extension of $H=\bigoplus(L /\langle g\rangle)$. Indeed, if this is not the case, we replace $K$ by the full subcategory $K^{\prime}$ of $B$ formed by all objects of $D$ and the objects $g^{m} y^{\prime}$ where $y^{\prime}$ is defined as follows. If $y$ is a source of $K$, then there exists a nonzero path (in R) $y \rightarrow a_{1} \rightarrow \cdots \rightarrow a_{t} \rightarrow a_{t+1} \rightarrow \cdots \rightarrow a_{s}=\nu^{-1} y$ such that $a_{1}, \ldots, a_{t} \in D$, $a_{t+1}, \ldots, a_{s} \notin D$, and we put $y^{\prime}=a_{t+1}$. If $y$ is a sink of $K$ then there exists a nonzero path $y \leftarrow a_{1} \leftarrow \cdots \leftarrow a_{t} \leftarrow a_{t+1} \leftarrow \cdots \leftarrow a_{s}=\nu y$ such that $a_{1}, \ldots, a_{t} \in D, a_{t+1}, \ldots, a_{s} \notin D$, and we put $y^{\prime}=a_{t+1}$. Consequently, $\bigoplus(K /\langle g\rangle)$ is a tubular extension of $H$ of tubular type $(p+1, q)$ or $(p, q+1)$. We shall prove now that this leads to a contradiction to the fact that $\mathscr{D}$ is a full translation subquiver of the component $\mathscr{C}$ and $D$ is the support category of $\mathscr{D}$. We use now the known description of the indecomposable preinjective modules over the tilted algebra $\bigoplus \Lambda$ of type $\widetilde{A}_{p, q}$ and the fact that $\mathscr{D} /\langle g\rangle$ is a full cofinite translation subquiver of the preinjective component of $\Gamma_{\Lambda}$. Then, for any $M(i, j)=M(L(i, j)) \in \mathscr{D}$ the line $L(i, j)$ is of the form

$$
e-\cdots \rightarrow a \leftarrow \cdots \leftarrow b \rightarrow \cdots \leftarrow c \rightarrow \cdots \rightarrow d \leftarrow \cdots-f
$$

where the line $a \leftarrow \cdots \leftarrow b \rightarrow \cdots \leftarrow c \rightarrow \cdots \rightarrow d$ is the common part of $L(i, j)$ and $L$, contains a line of the form $g^{k} x_{0}-\cdots-g^{k} y_{0}$, for some $k \in \mathbf{Z}, a$ and $d$ are not sinks of $L$, and may be $a=b, c=d, e=a$ or $d=f$. Assume now that $y$ is a source of $K$. Then there exists (up to symmetry) a line

$$
a \rightarrow \cdots-x \rightarrow z_{1} \leftarrow \cdots \leftarrow z_{t} \leftarrow y=y_{0}
$$

where $a$ is a source of $L, V: a \rightarrow \cdots-x$ is the support of a module $M \in \mathscr{D}$, $U: a \rightarrow-x \rightarrow z_{1} \leftarrow \cdots \leftarrow z_{t}$ is the support of a module $N \in \mathscr{D}$, and may be $t=1$.

Let $\Omega: z_{1} \leftarrow \cdots \leftarrow z_{T} \leftarrow y_{0} \leftarrow \cdots \leftarrow y_{s}$ be the maximal nonzero path in $R$ which is a $\nu$-slice of $R$, contains the path $z_{1} \leftarrow \cdots \leftarrow z_{t}$ and has $z_{1}$ as the end point. Obviously we have in $\mathscr{D}$ an arrow $N \rightarrow M$ and hence $V^{r}=U$. This is a contradiction to the existence of $\Omega$ and the definition of the operation ()$^{r}$.

Finally assume that $y$ is a sink of $K$. Then there exists (up to symmetry) a line $a \rightarrow \cdots-x \leftarrow z_{1} \rightarrow \cdots \rightarrow z_{t} \rightarrow y=y_{0}$ where $a$ is a source of $L, V: a \rightarrow \cdots-x$ is the support of a module $M \in \mathscr{D}, U: a \rightarrow \cdots-x \leftarrow$ $z_{1} \rightarrow \cdots \rightarrow z_{t}$ is the support of a module $N \in \mathscr{D}$, and may be $t=1$. Let $\Omega: z_{1} \rightarrow \cdots \rightarrow z_{t} \rightarrow y_{0} \rightarrow \cdots \rightarrow y_{s}$ be the maximal nonzero path in $R$ which is a $\nu$-slice of $R$, contains the path $z_{1} \rightarrow \cdots \rightarrow z_{t}$ and has $z_{1}$ as a source. Then there is an arrow $N \rightarrow M$ in $\mathscr{D}$ and hence $V^{r}=U$, again a contradiction, because of the existence of $\Omega$.

Therefore we proved that $R=\widehat{B}=\widehat{D}$. Now, since $\bigoplus(D /\langle g\rangle)$ is a tilted algebra of type $\widetilde{A}_{p, q}$, we deduce from $[\mathrm{S} 2,2.13]$ that $G^{\prime}=G /\langle g\rangle$ is an admissible infinite cyclic group of $K$-linear automorphisms of $\Lambda \cong D /\langle g\rangle$, and 
hence $G$ is a free abelian group of rank 2 (cf. [S2, 4.5]). Consequently, by [S2, 2.1] $A \cong \bigoplus(R / G) \cong \bigoplus\left(\widehat{\Lambda} / G^{\prime}\right)$ is representation-infinite and domestic.

2.4 Proposition. Let $A$ be a nondomestic special biserial self-injective algebra. Then $\Gamma_{A}$ has infinitely many (regular) components of the form $\mathbf{Z} A_{\infty}^{\infty}$.

Proof. We shall apply some arguments from [PS]. We know, by 1.5 , that $A \cong$ $\bigoplus(R / G)$ where $R$ is a simply connected special biserial self-injective category and $G$ is an admissible torsion-free group of $K$-linear automorphisms of $R$. Since $A$ is representation-infinite, we conclude as in [DS 5.1] that $R$ contains a full convex subcategory $L$ which is a $G$-periodic line. Observe that $L$ is a convex $\nu$-slice of $R$. For a convex $\nu$-slice $D$ of $R$, we denote (cf. [PS]) by $D^{+}$ (resp. $D^{-}$) the convex hull of the full subcategory of $R$ formed by the objects of $D$ and all objects $x$ of $R$ which satisfy the following two conditions:

(a) the $\langle\nu\rangle$-orbit of $x$ does not intersect $D$;

(b) $R(x, y) \neq 0$ (resp. $R(y, x) \neq 0$ ) for some object $y$ of $D$. It follows from [PS, Lemma 9] that $D^{+}$(resp. $D^{-}$) is a convex $\nu$-slice of $R$. Consider the following sequence of convex $\nu$-slices of $R, B_{0} \subset B_{1} \subset B_{2} \subset \cdots$ where, in the above notation, $B_{0}=L, B_{2 n-1}=B_{2 n-2}^{+}, B_{2 n}=B_{2 n-1}^{-}$for $n \geq 1$, and let $B=\bigcup_{n \in \mathbf{N}} B_{n}$. Then $B$ is a convex $\nu$-slice of $R$. Moreover, it was shown in [PS, §3] that $B$ is a gentle (locally bounded) tree category and $R=\widehat{B}$. Since $A \cong \bigoplus(\widehat{B} / G)$ is nondomestic, $B / G_{L}$ is not a (finite) branch extension of $L / G_{L}$ (otherwise $\bigoplus\left(B / G_{L}\right)$ is a tilted algebra of type $\widetilde{A}_{p, q}$ and $\bigoplus(\widehat{B} / G)$ is domestic) and hence $B \neq B_{n}$ for all $n \in \mathbf{N}$. Then $B$ contains a full subcategory $D$ of the form

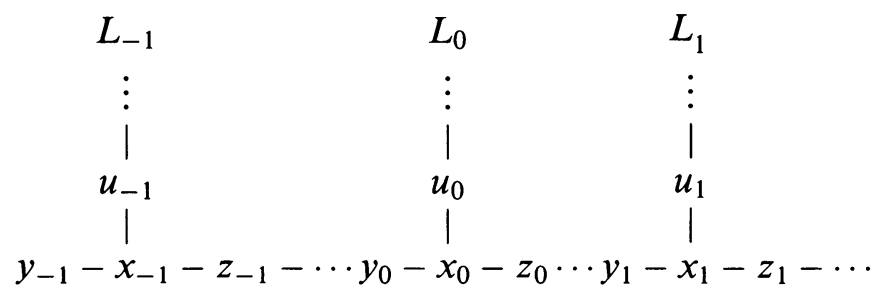

where the categories $L_{i}$ are lines of type $A_{\infty}$, and $g L_{i}=L_{i+1}, g x_{i}=x_{i+1}$, for all $i \in \mathbf{Z}$. Replacing, if necessary, $L_{i}$ by $L_{i}^{\prime}=g^{k} L_{i}$ and $x_{i}$ by $x_{i}^{\prime}=g^{k} x_{i}$ for a large $k \geq 1$, we may assume that the number of objects on the line $x_{0}-\cdots-x_{1}$ is greater than $\operatorname{dim}_{K} A$. By duality and symmetry, we may also assume that the arrows $u_{i}-x_{i}$ and $x_{i}-z_{i}$ are oriented as $u_{i} \rightarrow x_{i}$ and $x_{i} \rightarrow z_{i}$. Then $D$ is of the form

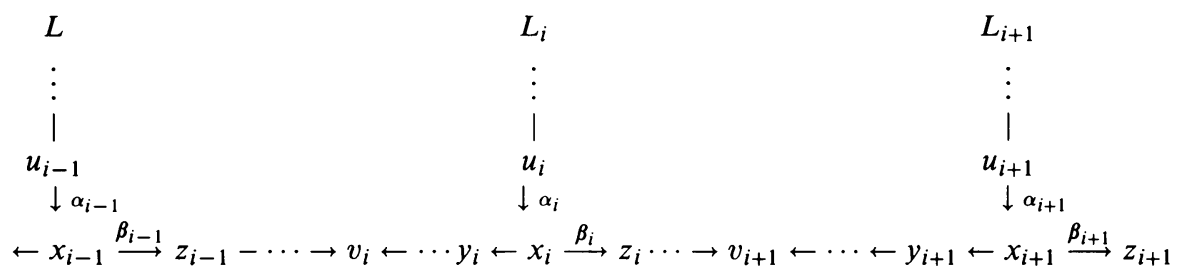

where $\alpha_{i} \beta_{i}=0$ and may be $v_{i}=x_{i}, i \in \mathbf{Z}$. Taking values of $\nu^{-1}$ at the source of the lines $L_{i}$ we conclude that $R=B$ contains lines $y_{i} \rightarrow \cdots \rightarrow v_{i} \rightarrow \cdots$ of type $A_{\infty}$ whose sources are the sinks of $L_{i}$ and the sinks are the values of $\nu^{-1}$ at the sources of $L_{i}$. For each $n \geq 1$, we denote by $M_{n}$ the $R$-module 
$M\left(W_{n}\right)$ given by the line

$$
W_{n}: v_{0} \leftarrow \cdots \leftarrow y_{0} \leftarrow x_{0} \rightarrow z_{0}-\cdots \rightarrow v_{n} \leftarrow \cdots \leftarrow y_{n} \leftarrow x_{n}
$$

and put $d_{n}=\operatorname{dim}_{K} M_{n}$. Observe that by our assumption on $D, d_{n}>\operatorname{dim}_{K} A$, and obviously $d_{n+1}>d_{n}$ for all $n \geq 1$. Let $\mathscr{C}_{n}$ denote the component of $\Gamma_{R}$ containing the module $M_{n}$. Observe that, for all $t, s \geq 0$, the lines $\left({ }^{t} W_{n}\right)^{r}={ }^{l}\left(W_{n}^{r^{s}}\right)$ contain the line $W_{n}$. Hence all predecessors of $M_{n}$ in $\mathscr{C}_{n}$ have dimension $\geq d_{n}$, and the Auslander-Reiten sequences terminating at these modules have two middle terms, because the radicals and the socle factors of indecomposable projective $R$-modules have dimension less than $\operatorname{dim}_{K} A$, and hence less than $d_{n}$. Similarly, all successors of $M_{n}$ in $\mathscr{C}_{n}$ have dimension $\geq d_{n}$, and the Auslander-Reiten sequences starting at these modules have two middle terms. Then, using properties of Auslander-Reiten sequences, we deduce that $\mathscr{C}_{n}$ is a regular component of the form $\mathbf{Z} A_{\infty}^{\infty}$, all modules in $\mathscr{C}_{n}$ have dimension $\geq d_{n}$ and that $M_{n}$ is the unique module in $\mathscr{C}_{n}$ of dimension $d_{n}$. Since $d_{1}<d_{2}<\cdots$ the components $\mathscr{C}_{n}$ are pairwise different. Then the images $\mathscr{C}_{n}^{\prime} \quad(n \geq 1)$ of the components $\mathscr{C}_{n}$ by the push-down functor $F_{\lambda}: \bmod R \rightarrow \bmod R / G$ are pairwise different regular components of $\Gamma_{R / G}$ with tree class $A_{\infty}^{\infty}$. Then, by [BR, §2] the components $\mathscr{C}_{n}^{\prime}$ are of the form $\mathbf{Z} A_{\infty}^{\infty}$. This finishes the proof of the proposition.

2.5 Proof of Theorem 2.1. The equivalence of (v), (vi), (vii) and (viii) is a direct consequence of [S2, 1.5, 2.13], of 1.5 and the fact that the Ringel's tubular algebras and tilted algebras of type $\widetilde{D}_{n}$ and $\widetilde{E}_{p}$ are not special biserial (cf. $[R 2, \S 5]$ ). The implications (iii) $\Rightarrow$ (ii), (iii) $\Rightarrow$ (i) and (iii) $\Rightarrow$ (iv) are trivial. Suppose (v) holds. In [ANS, 4.3], the AR-quiver of $\hat{\Lambda}$ is completely determined. It consists of infinitely many components of type $\mathbf{Z} \widetilde{A}_{p, q}$, tubes of rank $p, q$ and 1. From [S2, 2.13] we know the generators of $G$, and there is some $m$ such that $G$ has $m$ orbits on the components of each type. Therefore, by [DS, 2.5], the push-down functor $F_{\lambda}: \bmod \widehat{\Lambda} \rightarrow \bmod (\widehat{\Lambda} / G)$ is dense, and (iii) follows. Further, the implication (i) $\Rightarrow$ (vii) follows from Proposition 2.3 , and the implications (ii) $\Rightarrow$ (vii) and (iv) $\Rightarrow$ (vii) hold by Proposition 2.4.

2.6 Proof of Theorem 2.2. The implications (iii) $\Rightarrow$ (ii) and (ii) $\Rightarrow$ (i) are obvious. The equivalences (i) $\Leftrightarrow$ (v) and (iv) $\Leftrightarrow$ (v) follow from Theorem 2.1. We shall now show that (v) implies (iii). Assume that $A$ is not of polynomial growth. Let $A=\bigoplus(R / G)$ where $R$ is a simply connected self-injective locally bounded special biserial category and $G$ is an admissible torsion-free group of $K$-linear automorphisms of $R$. We know from 1.6 that

$$
\Gamma_{A} \cong \Gamma_{R / G} \cong\left(\Gamma_{R} / G\right) \amalg\left(\coprod_{L \in \mathscr{L}_{0}} \mathscr{T}_{L}\right)
$$

where $\mathscr{T}_{L}$ are $K^{*}$-families of stable tubes of rank 1. By [BR $\S 3$ ] there are only finitely many Auslander-Reiten sequences in $\bmod R / G$ with indecomposable middle term and terminating at the indecomposable $R / G$-modules of the first kind. Consequently, ${ }_{s} \Gamma_{A}$ does not contain components of the form $\mathbf{Z} A_{\infty}$ and contains at most finitely many components of the form $\mathbf{Z} A_{\infty} /\left\langle\tau^{n}\right\rangle$ with $n>1$. Moreover, we know by [HPR] that the components of ${ }_{5} \Gamma_{A}$ containing periodic 
modules are of the form $\mathbf{Z} A_{\infty} /\left\langle\tau^{m}\right\rangle, m \geq 1$. Let $\mathscr{C}$ be a component of ${ }_{s} \Gamma_{R / G}$ which does not contain periodic modules. Then, by [BR, §2] $\mathscr{C}=\mathbf{Z} \Delta / H$ for $\Delta=A_{\infty}^{\infty}$ and some admissible subgroup $H$ of $G$. If $H$ is nontrivial, then by [Z] $\mathscr{C}$ is of the form $\mathbf{Z} \mathscr{S}$ where $\mathscr{S}=\widetilde{A_{p}, q}$ for some $p, q \geq 1$, and consequently, by Proposition 2.3, $A \cong \bigoplus(R / G)$ is domestic, a contradiction to our assumption on $A$. Therefore $\mathscr{C}$ is of the form $\mathbf{Z} A_{\infty}^{\infty}$ and then (iii) is a consequence of Proposition 2.4.

This finishes the proof of the theorem.

\section{ON AUSLANDER-REITEN COMPONENTS FOR A CLASS OF SELF-INJECTIVE ALGEBRAS}

Let $A$ be a self-injective finite-dimensional algebra. We consider functions on the stable Auslander-Reiten quiver ${ }_{s} \Gamma_{A}$ of $A$.

For $A$-modules $X$ and $Y$, we denote by $\mathscr{P}_{A}(X, Y) \subseteq \operatorname{Hom}_{A}(X, Y)$ the subspace of maps which factor through a projective module, and by $\underline{\operatorname{Hom}}_{A}(X, Y)$ the quotient $\operatorname{Hom}_{A}(X, Y) / \mathscr{P}_{A}(X, Y)$.

3.1 Fix some $A$-module $W$, and define $d=d_{W}:{ }_{s} \Gamma_{A} \rightarrow \mathbf{N}$ by

$$
d_{W}(M)=\operatorname{dim}_{K} \underline{\operatorname{Hom}}_{A}(W, M) .
$$

3.2 Lemma. Suppose that $d=d_{W}$ as above. Assume that

(*) $0 \rightarrow \tau M \stackrel{j}{\rightarrow} E \stackrel{p}{\rightarrow} M \rightarrow 0$ is an Auslander-Reiten sequence.

(a) If $M$ is not a summand of $W$ then $d(M)+d(\tau M) \geq d(E)$.

(b) If in addition $\Omega M$ is not a summand of $W$ then equality holds in (a). In particular, if $\Delta$ is a component of ${ }_{s} \Gamma_{A}$ such that no indecomposable summand of $W$ belongs to $\Delta$ or $\Omega \Delta$ then $d_{W}$ is an additive function on $\Delta$.

This can be deduced from [AR2], using the fact that for self-injective algebras, $\Omega$ preserves AR-sequences [AR, VI]. For completeness, we include a (different) proof.

Proof. (a) Apply the functor $\operatorname{Hom}_{A}(W$,$) to (*)$. Since (*) is almost split and since $M$ is not a direct summand of $W$, we obtain an exact sequence

$$
0 \rightarrow(W, \tau M)_{A} \stackrel{j^{*}}{\longrightarrow}(W, E)_{A} \stackrel{p^{*}}{\longrightarrow}(W, M)_{A} \rightarrow 0 .
$$

Clearly, $j^{*}$ and $p^{*}$ preserve $\mathscr{P}_{A}($,$) . Moreover, if \eta \in \mathscr{P}_{A}(W, M)$ then there is some $\rho \in \mathscr{P}_{A}(W, E)$ with $p^{*}(\rho)=\eta$. Consequently we have a sequence

$$
0 \rightarrow \mathscr{P}_{A}(W, \tau M) \stackrel{j^{*}}{\longrightarrow} \mathscr{P}_{A}(W, E) \stackrel{p^{*}}{\longrightarrow} \mathscr{P}_{A}(W, M) \rightarrow 0
$$

which is exact at the ends, and $\operatorname{im} j^{*} \subseteq \operatorname{ker} p^{*}$. Therefore (a) follows.

(b) Suppose $\Omega M$ is not a summand of $W$. We have to show the following: $(* *)$ Let $\varphi: W \rightarrow \tau M$ such that $j \circ \varphi$ belongs to $\mathscr{P}_{A}(W, E)$. Then $\varphi \in \mathscr{P}_{A}(W, \tau M)$. Take such $\varphi$, and assume for contradiction that $\varphi \notin$ $\mathscr{P}_{A}(W, \tau M)$. Then there is an indecomposable summand, $W_{1}$ say, of $W$ such that $\varphi_{1}=\varphi_{W_{1}}$ does not belong to $\mathscr{P}_{A}\left(W_{1}, \tau M\right)$. Let $X$ be the push-out of $\varphi_{1}$ along an injective hull. That is, there is a commutative diagram with exact rows

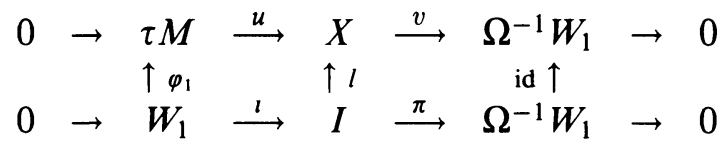


with $I$ injective. We will show that the top row is an almost split sequence. Then we are done since by the uniqueness of Auslander-Reiten sequences, $\Omega^{-1} W_{1} \cong M$, a contradiction to the hypothesis.

Explicitly, we may take $X=\tau M \oplus I / X_{0}$ where $X_{0}=\left\{\left(\varphi_{1} w, \imath w\right): w \in W_{1}\right\}$, and $u, v$ and $l$ are canonical maps.

First, we claim that $(* * *)$ does not split: Otherwise, there is some $\psi \in$ $\operatorname{Hom}_{A}(X, \tau M)$ such that $\psi u=\mathrm{id}_{\tau M}$ and we have that $\varphi_{1}=(\psi u) \varphi_{1}=$ $\psi\left(l_{l}\right)$ and $\varphi_{1}$ factors through $I$. Since $I$ is also projective, we have $\varphi_{1} \in$ $\mathscr{P}_{A}\left(W_{1}, \tau M\right)$, a contradiction.

Now let $\eta: \tau M \rightarrow Z$, and assume that $\eta$ is not a split monomorphism. Since $(*)$ is an Auslander-Reiten sequence, there exists some $\rho \in \operatorname{Hom}_{A}(E, Z)$ such that $\eta=\rho \circ j$. Now, $j \circ \varphi_{1}$ belongs to $\mathscr{P}_{A}\left(W_{1}, E\right)$ and $A$ is selfinjective, therefore $j \circ \varphi_{1}$ must factor through the injective hull $I$ of $W_{1}$. Let $\lambda \in \operatorname{Hom}_{A}(I, E)$ with $\lambda \circ l=j \circ \varphi_{1}$. Then we have a well-defined map $\rho: X \rightarrow Z$ given by $\rho\left[(m, a)+X_{0}\right]=\eta m-\rho \lambda a$, and moreover $\eta=\rho \circ u$. This shows that $(* * *)$ is almost split.

We wish to exploit functions of this form which we construct using approximate subalgebras.

3.3 Lemma. Let $B$ be a self-injective subalgebra of $A$ (with $1_{B}=1_{A}$ ). For $Y \in B$-mod and $M \in A-\bmod$ we have

(a) $\operatorname{Hom}_{B}(Y, M) \cong \operatorname{Hom}_{A}\left(A \otimes_{B} Y, M\right)$.

(b) If $A$ is projective as a $B$-module then this induces an isomorphism

$$
\underline{\operatorname{Hom}}_{B}(Y, M) \cong \underline{\operatorname{Hom}}_{A}\left(A \otimes_{B} Y, M\right) .
$$

3.4 Hypothesis. Let $A$ and $B$ be self-injective algebras, and suppose $\Delta$ is $a$ component of ${ }_{S} \Gamma_{A}$.

(1) $B$ is a subalgebra, such that $A$ is a projective $B$-module (and $1_{A}=1_{B}$ ).

(2) $B$ is local.

(3) For all $M$ in $\Delta, M$ is a direct summand of $A \otimes_{B} M$.

3.5 Let $W=A \otimes_{B} Y$ for some $B$-module $Y$, and consider $d=d_{W}$. Then $d_{W}(M)=d_{Y}\left(M_{B}\right)$. Moreover, write $M_{B}=M_{0} \oplus \sum B$, such that $M_{0}$ does not have a projective summand. Then $d(M)=d_{Y}\left(M_{0}\right)$.

We denote by $K$ the simple $B$-module.

3.6 Some remarks. (a) If $Y=K$ then $d_{Y}\left(M_{B}\right)=\operatorname{dim} \operatorname{soc}_{B}\left(M_{0}\right)$. In particular, if $M_{B}$ is not projective then $d_{Y}\left(M_{B}\right) \neq 0$ and $d_{A \otimes Y}(M) \neq 0$.

(b) If $Y \cong \tau Y$ then $d_{Y}$ is constant on $\tau$-orbits, hence induces a subadditive function on the tree of a component.

3.7 Lemma. Let $Y=K$. Suppose that $X$ is a $B$-module which does not have a projective summand. Then $\operatorname{dim}_{K} X \leq d_{Y}(X)(|B|-1)$.

Proof. Let $d=d_{Y}(X)$; then by 3.6(a), $d=\operatorname{dim} \operatorname{soc} X$. There is an injective hull $0 \rightarrow X \rightarrow P \stackrel{\pi}{\rightarrow} \Omega^{-1} X \rightarrow 0$ where $P=\sum \bigoplus_{d} B$. Moreover, $\pi(\operatorname{rad} P)=$ $\operatorname{rad} \Omega^{-1} X$ and therefore $\operatorname{dim} \Omega^{-1} X \geq d$. We deduce $d+\operatorname{dim} X \leq \operatorname{dim} \Omega^{-1} X+$ $\operatorname{dim} X=\operatorname{dim} P$, and the statement follows.

3.8 Proposition. Let $A, B$ be as in 3.4. Suppose that $\Delta$ is a Euclidean component of $A$. Let $\Psi=\left\{X \in\right.$ ind $B: A \otimes_{B} X$ has a summand in $\left.\Delta\right\}$. Then 
(a) One of $K$ or $\Omega^{-1} K$ belongs to $\Psi$.

(b) If $Y$ and $\Omega Y \in$ ind $B-\Psi$, where $Y$ is not periodic or projective we have that either $\underline{\operatorname{Hom}}_{B}(Y, \Delta)=0$ or $\operatorname{dim}\left\{\tau^{n} Y\right\}$ is bounded.

3.9 Remark. The last possibility, namely that there is a nonperiodic module such that the dimension of $\left\{\tau^{n} Y\right\}$ is bounded, does not arise for various types of components, in particular $A_{\infty}^{\infty}$ and $D_{\infty}$, see [MR].

Moreover, if $A$ is a group algebra or a block this never happens: In this case, $\tau \cong \Omega^{2}$. If $\operatorname{dim}\left\{\tau^{n} Y\right\}$ is bounded, then $Y$ has complexity $\leq 1$, in the sense of $[\mathrm{AE}]$. Then by the theorem of [AE], $Y$ is periodic.

Also, if $A$ is a tame self-injective algebra then by [CB1] for any nonperiodic module $Y$, the dimension of $\left\{\tau^{n} Y\right\}$ is unbounded.

Proof of 3.8. Suppose that (a) does not hold. Take $W:=A \otimes_{B} K$, then $d_{W}$ is additive on $\Delta$. Moreover, $d_{W}$ is nonzero on $\Delta$ : If $d_{W}(M)=\left|\underline{\operatorname{Hom}}_{B}(K, M)\right|=$ $\left|\operatorname{soc}_{B}\left(M_{0}\right)\right|=0$ then $M_{B}$ is projective. By the hypothesis (3) in 3.4 it follows that $M$ is projective; a contradiction.

In [HPR, Theorem (c), p. 286], the following is proved:

Suppose $\Sigma$ is a Euclidean diagram, then any additive function $\mathbf{Z} \Sigma \rightarrow \mathbf{N}_{0}$ is bounded. Hence $d_{W}$ takes bounded values on $\Delta$, by 3.2. So for any $M$ in $\Delta$ and for all $n \in \mathbf{Z}, d_{W}\left(\tau^{n} M\right)=\left|\underline{\operatorname{Hom}}_{B}\left(K, \tau^{n} M\right)\right| \leq$ a constant $C$. But this is equal to $\left|\operatorname{soc}_{B}\left(\tau^{n} M\right)_{0}\right|$. But then also $\operatorname{dim}\left(\tau^{n} M\right)_{0}$ is bounded, by 3.7. Using now the hypotheses (1) and (3) in 3.4 we deduce that also $\operatorname{dim}\left(\tau^{n} M\right)$ is bounded.

In [A], the following is proved: Let $R$ be a connected artinian ring with AR-sequences. If $R$ has an AR-component with modules of bounded length, then these are all indecomposable modules and $R$ is of finite representation type. Hence $\Delta$ is finite and $A$ is of finite type. This is a contradiction.

Since $\Omega(\Delta)$ is also Euclidean, we may assume that $K$ belongs to $\Psi$.

(b) Suppose now that the $B$-module $Y$ is nonperiodic and not projective, and that both $Y$ and $\Omega Y$ do not lie in $\Psi$. Consider the function $d_{W}$ where $W=A \otimes_{B} Y$. By 3.2, we know that $d_{W}$ is additive on $\Delta$, and consequently by [HPR, p. 286] (see above) $d_{W}$ must be bounded.

If $d_{W}=0$ then we have the first possibility. Otherwise, let $d_{W} \neq 0$. We have, taking $M=\tau^{-n} K$ that $\left|\underline{\operatorname{Hom}}_{B}\left(Y, \tau^{-n} K\right)\right|=\left|\underline{\operatorname{Hom}}_{B}\left(\tau^{n} Y, K\right)\right|=$ $\left|\operatorname{top}\left(\tau^{n} Y\right)_{0}\right| \leq$ a constant $C$. But then $\operatorname{dim} \tau^{n} Y$ is bounded.

3.10 Corollary. Let $A$ be local and self-injective. Suppose $A$ has infinitely many $\tau$-orbits of nonperiodic modules $Y$ with $\operatorname{dim}\left\{\tau^{n} Y\right\}$ unbounded. Then $A$ does not have a Euclidean component.

Proof. We take $A=B$; then the hypotheses in 3.4 are satisfied. Assume for contradiction that $A$ has a Euclidean component, $\Delta$ say. We apply the proposition. Here $\Psi$ is the set of modules in $\Delta$. Note that the component is fixed by $\Omega$ since $\Omega \Delta$ and $\Delta$ are both Euclidean, and there is only one projective module. Therefore $K$ belongs to $\Psi$. By the proposition and by the hypothesis, there is some $Y$ in ind $A-\Psi$ which is nonperiodic and for which $\operatorname{Hom}_{A}(Y, \Delta)=0$. Consequently $\underline{\operatorname{Hom}}_{A}(Y, K)=0$. On the other hand, $\underline{\operatorname{Hom}}_{A}(Y, K)=\operatorname{Hom}_{A}(Y, K)$ [since $Y$ is not projective and $K$ is simple]. Moreover, $K$ is the only simple module and therefore $Y=0$, a contradiction. 
As this result may suggest, we shall now prove that various algebras have infinitely many $\tau$-orbits of nonperiodic modules.

3.11 Lemma. Let $A$ be a local self-injective algebra, and let $m \in \mathbf{N}$ such that $\operatorname{soc}_{m}(A) \subseteq J^{2}$. Suppose $\left\{M_{n}\right\}$ is a sequence of nonperiodic indecomposable $A$ modules of unbounded dimension such that $J^{2} M_{n}=0$. If there is a $\tau$-periodic module $Z$ such that $J^{m} Z=0$, then the modules $M_{n}$ lie in infinitely many $\tau$-orbits.

Proof. Consider the function $d=d_{Z}$; then $d$ is additive on nonperiodic components and periodic on each $\tau$-orbit. We claim that $d\left(M_{n}\right) \geq \operatorname{dim} \operatorname{soc} M_{n}$; this implies that the numbers $d\left(M_{n}\right)$ are unbounded; therefore the modules must belong to infinitely many $\tau$-orbits.

Observe first that $\mathscr{P}_{A}\left(Z, M_{n}\right)=0$ : Suppose $\varphi \in \mathscr{P}_{A}\left(Z, M_{n}\right)$; since $A$ is self-injective, $\varphi$ factors through an injective hull of $Z$; hence there is a commutative diagram

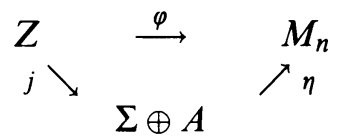

Since $J^{m} Z=0$ we have that $j(Z) \subseteq \operatorname{soc}_{m}[\Sigma \oplus A] \subseteq \Sigma \oplus J^{2}$. Consequently $\eta j(Z) \subseteq \eta\left(\Sigma \oplus J^{2}\right) \subseteq J^{2}[\operatorname{im} \varphi]=0$, and therefore $d_{Z}(M)=\operatorname{dim}_{H_{0}}\left(Z, M_{n}\right)$ $\geq \operatorname{dim} \operatorname{soc} M_{n}$.

3.12 Corollary. Let $\Lambda$ be the local algebra

$$
\Lambda=K\langle\alpha, \beta\rangle /\left(\beta^{2}, \alpha^{2}-(\beta \alpha)^{m} \beta,(\alpha \beta)^{m}-(\beta \alpha)^{m}, \alpha^{3}\right), \quad m \geq 1 .
$$

Then $\Lambda$ does not have a Euclidean component.

Proof. We apply 3.11. Let $M_{n}$ be the string module of dimension $2 n+1$ :

$$
K \stackrel{\alpha}{\leftarrow} K \stackrel{\beta}{\rightarrow} K \leftarrow \cdots \leftarrow K \stackrel{\beta}{\rightarrow} K .
$$

We take for $Z$ a 2-dimensional module

$$
Z=K \underset{\alpha=1}{\stackrel{\beta=\lambda}{\rightrightarrows}} K \quad(\lambda \neq 0) \text {. }
$$

Then $\tau Z \cong Z$ (see [CB2]). Now we have to show

(1) $M_{n}$ is not periodic.

Then it follows from 3.11 that the $M_{n}$ lie in infinitely many $\tau$-orbits. Moreover, the corollary is proved by 3.10 if we establish that

(2) If $Y=M_{n}$ then the dimension of $\left\{\tau^{k} Y\right\}$ is unbounded.

Let $\theta$ be the component of $M_{n}$. Consider the module $W=A \beta$; then $\tau W \cong W \cong \Omega W$. It has been proved in [E2] that $d_{W}(M)=1$ or 2 for arbitrary string modules $M$. Moreover, all modules in the component of $W$ are asymmetric strings whereas $M_{n}$ is symmetric, in the notation of [CB2]. So $W$ does not belong to $\theta$, and $d_{W}$ is a nonzero bounded additive function on $\theta$. It induces a bounded additive function on the tree of $\theta$.

Consequently $\theta$ is not a tube, and (1) follows. Moreover, by [HPR], the tree class of $\theta$ must be either $A_{\infty}^{\infty}$ or $D_{\infty}$ (or Euclidean), and then (2) follows from [MR] (or from Auslander's Theorem [A] (see the proof of 3.8)).

3.13 Lemma. Let $A=K G$ where $G$ is a p-group and char $K=p$. Suppose $G$ is noncyclic and not a Klein 4-group or a quaternion group. Then $A$ has 
infinitely many $\tau$-orbits of nonperiodic modules. In particular, $A$ does not have a Euclidean component.

This follows also from [L, Lemma 3.1] and for $p>2$ from [O, Lemma 4]. We include a (different) proof since it is elementary and exploits the same principles as 3.12 .

We will use the following well-known facts from the theory of modular group representations:

3.13.1 Suppose $G$ is a $p$-group and $\operatorname{char} K=p$.

(a) Let $Q$ be a subgroup of $G$. If $M$ is an indecomposable $K Q$-module then $K G \otimes_{K Q} M$ is indecomposable [Green's theorem, see e.g. [Be]].

(b) If $Y$ is a periodic $K G$-module and $G$ is not cyclic or quaternion then $\operatorname{dim}_{K} Y$ is divisible by $p$ (see [C]).

Proof of 3.13. By Green's theorem it suffices to prove the lemma for a group $G$ such that $|G|$ is minimal subject to the hypothesis. Then by elementary group theory $G$ is either dihedral of order 8 or $C_{2} \times C_{4}$ or $C_{p} \times C_{p}$ for $p>2$. We apply 3.11 .

In all cases, the group algebra is $\cong K\langle\alpha, \beta\rangle / I$ for an appropriate (wellknown) ideal. Take $M_{n}$ to be

$$
K \stackrel{\alpha}{\leftarrow} K \stackrel{\beta}{\rightarrow} K \stackrel{\alpha}{\leftarrow} \ldots \stackrel{\beta}{\rightarrow} K
$$

of dimension $2 n+1$. Then $M_{n}$ is not periodic, by 3.13.1 if we take those $n$ where $p$ does not divide $2 n+1$. For $Z$, take a periodic module of dimension $p$.

If $p=2$ one may take

$$
Z=K \underset{\beta=1}{\stackrel{\alpha=\lambda}{\rightrightarrows}} K \quad \text { with } \lambda \neq 0 .
$$

Then it is easily verified that $Z \cong \Omega^{2} Z$ [which is also well known in the dihedral case, since then $K G$ is special biserial, see [BR] or Chapter 1]. If $p>2$ we take $Z=A \alpha^{p-1}$. In all cases, it is easy to check that $Z \subseteq \operatorname{soc}_{p} A$, and the statement follows from 3.10.

We note the following consequence:

3.13.2 Corollary. Let $D$ be a p-block of some group algebra $K G$ with defect group $P$. If $P$ is not cyclic or a Klein 4-group of a generalized quaternion group then $D$ has infinitely many $\tau$-orbits of nonperiodic modules with a given vertex $Q \leq P$ where $Q$ is not cyclic or a Klein 4-group of generalized quaternion.

Proof. By the Brauer correspondence of blocks and the Green correspondence for module which preserves $\tau$, we may assume that $P$ is normal in $G$. The statement follows then from 3.13 by standard arguments.

We shall now study Euclidean components for blocks. Let $D$ be a block, that is $D$ is an indecomposable algebra which is a direct summand of some group algebra.

A defect group of $D$ may be defined as a minimal subgroup $P$ of $G$ such that every $D$-module $M$ is a direct summand of $K G \otimes_{K P} M$. It is well known that any such $P$ is a $p$-group and is unique up to $G$-conjugation. In particular, with $A=K G$ and $B=K P$ the hypotheses in 3.4 are satisfied, for any component of ${ }_{s} \Gamma_{D}$. 
A vertex of an indecomposable $K G$-module $M$ is a minimal subgroup $V$ of $G$ such that $M$ is a direct summand of $K G \otimes_{K V} S$ for some indecomposable $K V$-module $S$. In this case, $S$ is a source of $M$. Also, $V$ is a $p$-group and is unique up to $G$-conjugation. Moreover, any two sources of $M$ are conjugate in $N_{G}(V)$.

We recall that a module whose vertex is cyclic or generalized quaternion must be periodic; this follows from [AE].

Let $H$ be a subgroup of $G$, we use the notation $K_{H}^{G}$ for the module $K G \otimes_{K H} K$.

3.14 Theorem. Let $D$ be a block of a group algebra $K G$ with defect group $P$. Then $D$ does not have a Euclidean component unless $p=2$ and $P$ is a Klein 4-group.

Proof. Assume that $D$ has a Euclidean component, $\Delta$ say. Take $A=K G$ and $B=K P$; then the hypotheses in 3.4 are satisfied, and we apply the proposition. We may assume that $K$ belongs to $\Psi$ [otherwise we replace $\Delta$ by $\Omega^{-1} \Delta$ ]; and write $K^{G}=M \oplus M^{\prime}$ with $M$ in $\Delta$.

The module $M_{P}$ is a direct sum of modules of the form $K_{P^{x} \cap P}^{P}$ for $x \in G$; this follows from the Mackey decomposition and Green's theorem [see 3.13.1].

By 3.4(3) there is some $Q=P^{x} \cap P$ such that $M$ is a summand of $K_{Q}^{G}$. Fix such $Q$; note that $Q$ is not cyclic or generalized quaternion. Put $M_{1}:=K_{Q}^{P}$, and remember that $M_{1}$ is a summand of $M_{P}$. Now suppose that $Y \in B$-mod is nonperiodic and not projective.

(1) If $Y$ and $\Omega$ do not belong to $\Psi$ then $Y_{Q}$ is projective. By 3.8 and 3.9 we have that $\underline{\operatorname{Hom}}_{B}(Y, \Delta)=0$. In particular, $\underline{\operatorname{Hom}}_{B}(Y, M)=0$ and then $0=\underline{\operatorname{Hom}}_{B}\left(Y, M_{1}\right) \cong \underline{\operatorname{Hom}}_{K Q}\left(Y, K_{Q}\right)$, by the Nakayama relations for group representations. Now, $K_{Q}$ is the only simple $K Q$-module, and therefore $Y_{Q}$ must be projective.

As we noted above, $Q$ must contain a subgroup $V \cong C_{p} \times C_{p}$.

(2) We may assume that $Q=V$. We will show that there is a module $Z$ in $\Delta$ with vertex $V$ and source $K_{V}$. Let $Y=K_{V}^{P}$; then $Y_{Q}$ is not projective and therefore $Y$ belongs to $\Psi$. Choose a summand $N$ of $Y^{G}$ which lies in $\Delta$. As in (1), a source of $N$ in $P$ is of the form $K_{V^{x} \cap P}^{P}$. Since $N$ is not periodic, the group $V^{x} \cap P$ is not cyclic and is therefore $=V^{x}$ which is $G$-conjugate to $V$.

Then $V$ is necessarily a minimal vertex of the modules in $\Delta$. Now let $\Psi_{0}=\{Y \in \Psi: \operatorname{vertex}(Y)=V\}$. Then $\Psi_{0}$ is closed under $\tau$. We claim that $\Psi_{0}$ contains only finitely many $\tau$-orbits: Let $N$ be in $\Delta$, and suppose there is some $N_{0}$ in $\Psi_{0}$ such that $N$ is a summand of $N_{0}^{G}$. Then it follows that $\operatorname{vertex}(N)=V$, and a source of $N_{0}$ is a source of $N$. As we noted above, the number of sources is finite.

By (1) and (2), all nonperiodic $P$-modules with vertex $V$ belong to $\Psi_{0}$. Hence $V$ has only a finite number of $\tau$-orbits of nonperiodic modules. We deduce from Lemma 3.13 that $V$ is a Klein 4-group; in particular $p=2$.

It remains to show that $P=V$.

(3) We may assume that $V$ is a normal subgroup of $G$. Let $A_{0}$ be the block of $N_{G}(V)$ containing the Green correspondent of some $M$ in $\Delta$ with vertex $V$. By the theorem of Kawata [K] which we will state below in 3.15, $A_{0}$ must have a Euclidean component. If we show that the defect group of $A_{0}$ is $V$ then it follows form general block theory (see [AB]) that $V$ is also a defect group of $D$. 
Let $\Delta=\Delta \cup \Omega \Delta$. Our aim is to show that all simple $D$-modules belong to $\Delta$. To do this, consider the set of modules $\mathscr{V}=\{M \in$ ind $D: M$ is a summand of $\left.K_{V}^{G}\right\}$. We will use properties listed below in 3.16 .

By (1) and (2), there is some $M$ in $\mathscr{V}$ which belongs to $\Delta$. Choose such $M=M_{0}$, with socle $S_{0}$ (see $3.16($ a) $)$.

(4) If $M_{0} \in \mathscr{V} \cap \Delta$ and $S_{0}=\operatorname{soc} M_{0}$ then $S_{0} \in \Delta$ :

Suppose not, then $d_{S_{0}}$ is an additive function on $\Delta$ which is bounded on $\Delta$. On the other hand, it follows from 3.16(e) that $\operatorname{soc}_{V}\left(\tau^{n} M_{0}\right) \cong \sum \bigoplus d_{i} M_{i}$; moreover the multiplicity of $M_{0}$ as a summand is unbounded for $n \rightarrow \infty$. Consequently the dimension of $\operatorname{Hom}_{A}\left(S_{0}, \tau^{n} M_{0}\right)$ is unbounded as well. But this is equal to $\left|\underline{\operatorname{Hom}}_{A}\left(S_{0}, \tau^{n} M_{0}\right)\right|$ since $S_{0}$ is simple. Therefore $d_{S_{0}}$ is unbounded, a contradiction.

Now assume $S_{1}$ is simple and $\operatorname{Ext}^{1}\left(S_{1}, S_{0}\right) \neq 0$. Assume first that $S_{1}$ is a composition factor of $M_{0}$. By (4) it suffices to show that $M_{1}$ belongs to $\Delta$.

Suppose not, then $d_{M_{1}}$ is additive on $\Delta$ and hence is bounded. On the other hand, $d_{M_{1}}\left(\tau^{n} M_{0}\right)=\left|\underline{\operatorname{Hom}}_{A}\left(M_{1}, \tau^{n} M_{0}\right)\right|=\left|\operatorname{Hom}_{A}\left(M_{1}, \tau^{n} M_{0}\right)\right|$, by 3.16(c), and this is $\geq$ the multiplicity of $M_{0}$ as a summand of $\operatorname{soc}_{V}\left(\tau^{n} M_{0}\right)$, by $3.16(\mathrm{~b})$. we have observed in the proof of (4) that this number is unbounded as $n \rightarrow \infty$.

Otherwise, $S_{1} \subseteq \operatorname{soc} \Omega^{-1} M_{0}$. By 3.16, we have again that $\operatorname{soc}_{V}\left[\tau^{n} \Omega^{-1} M_{0}\right]=$ $\sum \bigoplus d_{i} M_{i}$. Since $S_{1} \subseteq \operatorname{soc} \Omega^{-1} M_{0}$, we deduce that $M_{1}$ occurs for $n=0$, and then by 3.16(b) and (e), the multiplicity of $M_{1}$ is unbounded as $n \rightarrow \infty$. One shows now as before that $M_{1}$ belongs to $\Delta$ and then $S_{1}$ as well, by (4).

The quiver of $D$ is connected, therefore all simple modules belong to $\Delta$. We will now show that all nonperiodic indecomposable $D$-modules must belong to $\Delta$.

Let $Z$ be such a module, and consider $d_{Z}$. Put $X=\sum \bigoplus S_{i}$, the sum of all simple $A$-modules. Then $d_{Z}\left(\tau^{-n} X\right)=\left|\underline{\operatorname{Hom}}_{A}\left(Z, \tau^{-n} X\right)\right|=\left|\underline{\operatorname{Hom}}_{A}\left(\tau^{n} Z, X\right)\right|$ $=\left|\operatorname{Hom}_{A}\left(\tau^{n} Z, X\right)\right|$. If $d_{Z}$ were additive on $\Delta$ or $\Omega \Delta$ then it would follow that the length of top $\tau^{n} Z$ is bounded, and then $\operatorname{dim} \tau^{n} Z$ as well, a contradiction to 3.9 .

Hence $D$ has only finitely many $\tau$-orbits of nonperiodic modules. By 3.13.2, $P$ is a Klein 4-group.

3.15 Theorem (Kawata [K]). Suppose $\Delta$ is a component of the stable AuslanderReiten quiver of $K G$. Let $V$ be a minimal vertex of the modules in $\Delta$ and $\mathscr{C}$ the component of ${ }_{s} \Gamma_{\Lambda}$ containing the Green correspondent of some module $M$ in $\Delta$ with vertex $V$ where $\Lambda=K\left[N_{G}(V)\right]$. Then there is a subquiver $\mathscr{C}_{0}$ of $\mathscr{C}$ and a graph isomorphism $\mathscr{C}_{0} \rightarrow \Delta$.

It remains to summarize the properties of the modules in $\mathscr{V}$ we used.

3.16 Let $G$ be a finite group and $V \unlhd G$ a Klein 4-group. Suppose $K$ is an algebraically closed field of characteristic 2 , and that $D$ is a block of $A=K G$. Define $\mathscr{V}=\left\{M \in\right.$ ind $D: M$ is a summand of $\left.K_{V}^{G}\right\}$.

(a) $\operatorname{soc} M \cong \operatorname{top} M$ and is simple; moreover each simple $A$-module occurs exactly once as a top factor of some $M \in \mathscr{V}$. We denote by $M_{i}$ the module

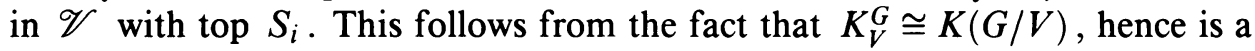
group algebra, which also implies that

(b) $\left|\operatorname{Hom}_{A}\left(M_{i}, M_{j}\right)\right|$ is equal to the multiplicity of $S_{i}$ as a composition factor of $M_{j}$. 
(c) If $X \in D$-mod such that $X_{V}$ does not have a projective summand and $M \in \mathscr{V}$ then $\mathscr{P}_{A}(M, X)=0=\mathscr{P}_{A}(X, M)$. This follows easily from the fact that $V$ acts trivially on $M$.

The following properties may be proved by inducing the $K V$-modules $\Omega^{n}(K)$ which are $G$-stable, and studying their indecomposable direct summands, using for example [HB, §9].

(d) Suppose $P_{i}$ is an indecomposable projective $D$-module with top $S_{i}$.Then

$$
0 \subset M_{i} \subset \Omega M_{i} \subset P_{i} \text { and } \Omega M_{i} / M_{i} \cong M_{j} \oplus M_{k}
$$

for some $M_{j}, M_{k}$ in $\mathscr{V}$. Moreover, the multiplicity of $M_{j}$ as a summand of $\Omega M_{i} / M_{i}$ is the same as the multiplicity of $M_{i}$ as a summand of $\Omega M_{j} / M_{j}$.

This allows to construct a special biserial algebra associated to $D$ and $\mathscr{V}$. We define a quiver where the vertices are the simple $D$-modules. The number of arrows $i \rightarrow j$ is defined to be the multiplicity of $M_{j}$ as a direct summand of $\Omega M_{i} / M_{i}$.

Then, by (d), we have that at each vertex $i$, two arrows start and two arrows end. Moreover, the number of arrows $i \rightarrow j$ is the same as the number of arrows $j \rightarrow i$. Any connected component has at most three vertices, since it arises from inducing simple $C(V)$-modules to $G$ and $G / C(V)$ is isomorphic to a subgroup of $S_{3} \quad(=$ Aut $V)$. In fact, this implies that the connected components of the quiver are of the form:

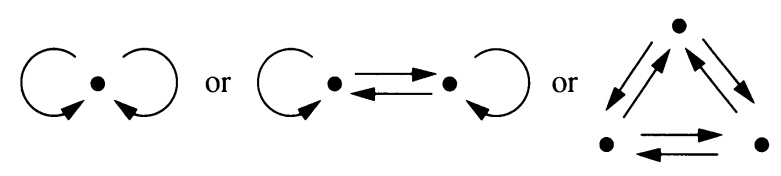

We define now $\mathscr{S}_{D}$ to be the symmetric special biserial algebra with this quiver such that $J^{3}=0$; and we denote by $\mathbf{S}_{i}$ the simple $\mathscr{S}_{D}$-module corresponding to the vertex $S_{i}$. We will now describe $\Omega^{n} M$ for all $M$ in $\mathscr{V}$ and $n \in \mathbf{Z}$.

(e) The submodule $\operatorname{soc}_{V}\left(\Omega^{n} M\right)$ is a direct sum of modules $M_{j}$ in $\mathscr{V}$ and so is the quotient $\Omega^{n} M / \operatorname{soc}_{V}\left(\Omega^{n} M\right)$. Suppose $M=M_{0}$; then the structure of the module $\Omega^{n} \mathbf{S}_{0}$ of the algebra $\mathscr{S}_{D}$ describes which $M_{j}$ occur in this filtration of $\Omega^{n} M$ and how they extend.

In particular, if $\mathbf{S}_{0}$ and $\mathbf{S}_{j}$ belong to the same connected component of the quiver then the number of $M_{j}$ in $\operatorname{soc}_{V}\left(\Omega^{2 n} M_{0}\right)$ or in $\operatorname{soc}_{V}\left(\Omega^{2 n+1} M_{0}\right)$ is unbounded as $n \rightarrow \infty$.

\section{SOME APPLICATIONS}

Let $K$ be an algebraically closed field with char $K=p>0, G$ a finite group and $B$ be a block of $K G$. Concerning Euclidean components, we summarize:

4.1 Theorem. The following conditions are equivalent: $\Delta$.

(i) ${ }_{s} \Gamma_{B}$ has a connected component of the form $\mathrm{Z} \Delta$ for some Euclidean quiver

(ii) ${ }_{s} \Gamma_{B}$ has a connected component of the form $\mathbf{Z} \tilde{A}_{1,1}$ or $\mathbf{Z} \tilde{A}_{3,3}$.

(iii) $p=2$ and the defect groups of $B$ are Klein 4-groups.

(iv) $B$ is Morita equivalent to one of the algebras given by the following quiver 
and relations:

$$
\begin{aligned}
& B_{1}: \alpha \bigcirc \alpha^{2}=\beta^{2}=0, \quad \alpha \beta=\beta \alpha . \\
& B_{2}: \bullet \stackrel{\beta}{\underset{\gamma}{\rightleftarrows}} \cdot \stackrel{\delta}{\underset{\eta}{\rightleftarrows}} \bullet \quad \beta \gamma=\eta \delta=0, \quad \gamma \beta \delta \eta=\delta \eta \gamma \beta .
\end{aligned}
$$

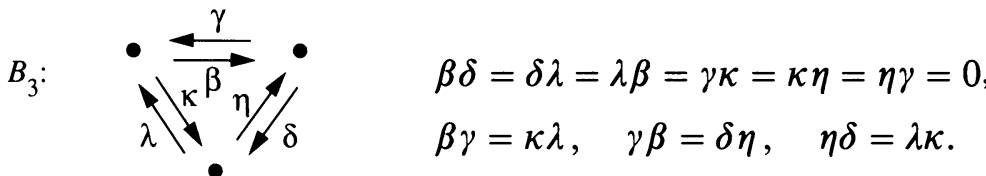

(v) $B$ is one-parametric.

(vi) $B$ is representation-infinite of polynomial growth.

This follows from 3.14 and the results of [E1] or the lists in [E2, pp. 294-297].

Remarks. (1) If $B=B_{1}$ then ${ }_{s} \Gamma_{B}$ consists of one component of the form $\mathrm{Z} \widetilde{A}_{1,1}$ and one $P_{1}(K)$-family of tubes of rank 1 .

(2) If $B=B_{2}$ or $B=B_{3}$ then ${ }_{s} \Gamma_{B}$ consists of one component of the form $\mathbf{Z}{\widetilde{A_{3}, 3}}$, two tubes of rank 3 and one $K^{*}$-family of tubes of rank 1 .

(3) For small fields, it is also possible that $\mathscr{C} \cong \mathbf{Z} \widetilde{B}_{3}$, see [Be, Bs]. This occurs if and only if the block has two simple modules.

For the tree class of stable components for ${ }_{s} \Gamma_{B}$, this means

4.2 Corollary. The following conditions are equivalent:

(i) ${ }_{5} \Gamma_{B}$ has a connected component whose tree class is a Euclidean tree.

(ii) ${ }_{S} \Gamma_{B}$ has a connected component whose tree class is of the form $\stackrel{(2,2)}{-} \cdot$.

(iii) $p=2$ and $B$ is Morita equivalent to $K V_{4}$ (the group algebra of the Klein 4-group).

4.3 Corollary. Assume that $B$ is representation-infinite and $B$ is not Morita equivalent to $\mathrm{KV}_{4}$ in characteristic 2 . Then the tree class of any component of ${ }_{5} \Gamma_{B}$ is one of the trees $A_{\infty}, D_{\infty}$ or $A_{\infty}^{\infty}$.

4.4 Corollary. Assume that the defect groups of $B$ are neither cyclic nor Klein 4-groups. Then any component of ${ }_{s} \Gamma_{B}$ is of the form $\mathbf{Z} A_{\infty}, \mathbf{Z} A_{\infty} /\left\langle\tau^{n}\right\rangle, \mathbf{Z} D_{\infty}$ or $\mathrm{Z} A_{\infty}^{\infty}$.

For tame blocks $B$ which have nonperiodic modules we have now precise information about the graph structure of ${ }_{s} \Gamma_{B}$.

4.5 Corollary. Let $p=2$ and suppose that $B$ is a block whose defect groups are dihedral of order $\geq 8$. Then ${ }_{s} \Gamma_{B}$ is a disjoint union of at most two tubes of rank 3, infinitely many tubes of rank 1 and infinitely many components of the form $\mathbf{Z} A_{\infty}^{\infty}$.

By [E1; E2, VI.10.1], $B / \operatorname{soc} B$ is special biserial, and the stable AR-quiver consists of components of the form $\mathrm{Z} A_{\infty}^{\infty} / \Pi$ for some group $\Pi$ and otherwise 
of tubes of rank 1, 3; and moreover the number of 3-tubes is at most two. Moreover, by $3.14, s^{\Gamma} B$ does not have a Euclidean component. Now the Corollary follows from the results of Chapter 2.

4.6 Corollary. Let $p=2$, and suppose that the defect groups of $B$ are semidihedral. Then ${ }_{s} \Gamma_{B}$ is a disjoint union of at most one tube of rank 3 and infinitely many components of each of the following types: 1-tubes, 2-tubes, $\mathbf{Z} A_{\infty}^{\infty}$ and $\mathbf{Z} D_{\infty}$.

By 3.14, we know now that $B$ does not have a Euclidean component. The rest has been proved in [E2, II.10].

In [E1, E2], algebras of dihedral type have been studied and classified. By definition, an algebra $\Lambda$ is of dihedral type if it is connected, symmetric and the Cartan matrix of $\Lambda$ is nonsingular, and moreover ${ }_{s} \Gamma_{\Lambda}$ is a disjoint union of

(i) tubes of rank 1 or 3, and the number of 3-tubes is at most 2.

(ii) nonperiodic components of tree class $A_{\infty}^{\infty}$ or $\widetilde{A}_{1,2}$.

It has been proved in [E1; E2, VI.10.1] that for any such algebra $\Lambda$, the socle factor is special biserial. However, it was not clear when Euclidean components occur. Now it is easy to answer this question.

4.7 Corollary. Let $\Lambda$ be an algebra of dihedral type. Then $\Lambda$ is of polynomial growth if and only if $\Lambda$ is one of the following.

$D(1)$

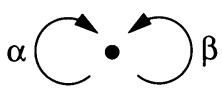

$D(2 . t)$

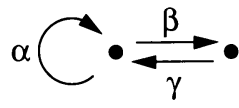

$D(3 . \not)$

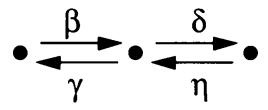

$D(3 . \Re)_{2}$

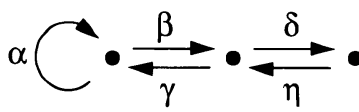

$D(3 \mathscr{L})_{2}$

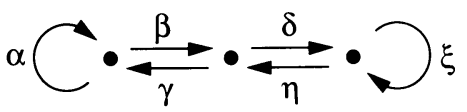

$$
\begin{aligned}
& \gamma \alpha=\alpha \beta=\beta \delta=\eta \gamma=\delta \xi=\xi \eta=0, \gamma \beta=\delta \eta \\
& \beta \gamma=\alpha^{2}, \eta \delta=\xi^{2} .
\end{aligned}
$$


$D(3 . \mathscr{K})$

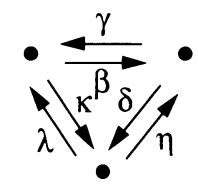

$$
\begin{aligned}
& \beta \delta=\delta \lambda=\lambda \beta=\gamma \kappa=\kappa \eta=\eta \gamma=0, \\
& \beta \gamma=\kappa \lambda, \quad \gamma \beta=\delta \eta, \quad \eta \delta=\lambda \kappa .
\end{aligned}
$$

$D(3 \%)$

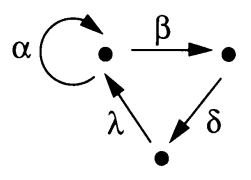

$$
\alpha \beta=0=\lambda \alpha,(\beta \delta \lambda)^{2}=\alpha^{2},(\delta \lambda \beta)^{2} \delta=0
$$

$D(3 /)$

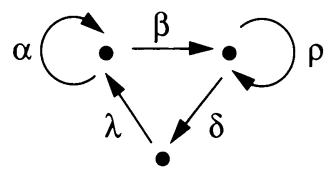

$$
\begin{aligned}
& \alpha \beta=\lambda \alpha=\beta \rho=\rho \delta=0 \\
& \beta \delta \lambda=\alpha^{2}, \delta \lambda \beta=\rho^{2} .
\end{aligned}
$$

Otherwise, ${ }_{s} \Gamma_{\Lambda}$ has no Euclidean components.

The algebras $D(1)(b)$ and $D(2 \mathscr{A})(b)$ are not special biserial but they have the same socle factors as $D(1)(a)$ and $D(2 \mathscr{A})(a)$, and hence they have the same Auslander-Reiten quiver and we can apply the results of Chapter 2. Note that the algebras of 4.1 occur.

4.8 Corollary. Let $\Lambda$ be an algebra of dihedral type. Then $\Lambda$ is of polynomial growth if and only if ${ }_{s} \Gamma_{\Lambda_{\widetilde{C}}}$ consists of one of the following:

(i) One component $\mathbf{Z} \widetilde{A_{1}, 1}$ and infinitely many 1-tubes.

(ii) One component $\mathbf{Z} \widetilde{A_{1}, 3}$, one 3-tube and infinitely many 1-tubes.

(iii) One component $\mathbf{Z} \widetilde{A_{3}, 3}$, two 3-tubes and infinitely many 1-tubes.

4.9 Corollary. Let $\Lambda$ be a tame local symmetric algebra. Then $\Lambda$ has a Euclidean component if and only if $\operatorname{dim} \Lambda=4$.

Proof. In [E2] we have obtained a list of these algebras; by short cuts of [R]. It turns out that any tame local symmetric algebra is either of dihedral type or of semidihedral type or of quaternion type, as defined in [E2].

If $\Lambda$ is of dihedral type then $\Lambda$ has a Euclidean component if and only if $\Lambda$ is 4-dimensional, as we have just seen.

Suppose $\Lambda$ is of semidihedral type. Then $\Lambda / \operatorname{soc} \Lambda \cong \Lambda_{m} / \operatorname{soc} \Lambda_{m}$ where $\Lambda_{m}$ is the algebra in 3.12; and we have proved that $\Lambda$ does not have a Euclidean component. Also, $\operatorname{dim} \Lambda \geq 8$.

Now assume that $\Lambda$ is of quaternion type, then $\Omega^{4} K \cong K$. It follows that all indecomposable modules have complexity $\leq 1$, hence their $\tau$-orbits have bounded dimension. If there were a Euclidean component $\Delta$ say then it would follow that all modules in $\Delta$ have bounded dimension. This is a contradiction to Auslander's theorem. 


\section{ACKNOWLEDGMENT}

The authors were supported by the DFG-Projekt Darstellungstheorie at the Universities of Essen and Bielefeld. The second author also wishes to thank the University of Oxford for the hospitality and support and the Polish Ministry of Education for its support under Research Project R.P.I.10. The authors are grateful to T. Okuyama for a useful discussion.

\section{REFERENCES}

[AB] J. L. Alperin and M. Broué, Local methods in block theory, Ann. of Math. (2) 110 (1979), 143-157.

[AE] J. L. Alperin and L. Evens, Representations, resolutions and Quillen's dimension theorem, J. Pure Appl. Algebra 22 (1981), 1-9.

[AS1] I. Assem and A. Skowroński, Iterated tilted algebras of type $\widetilde{A}_{n}$, Math. Z. 195 (1987), 269-290.

[AS2] _ On some classes of simply connected algebras, Proc. London Math. Soc. 56 (1988), 417-450.

[ANS] I. Assem, J. Nehring and A. Skowroński, Domestic trivial extensions of simply connected algebras, Tsukuba J. Math. 13 (1989), 31-72.

[A] M. Auslander, Applications of morphisms determined by objects, Proc. Conf. on Representation Theory (Philadelphia, 1976), Marcel Dekker, 1978, pp. 245-327.

[AR] M. Auslander and I. Reiten, Representation theory of Artin algebras. III, IV and VI, Comm. Algebra 3 (1975), 239-294; 5 (1977), 443-518; 6 (1978), 257-300.

[AR2] _ Stable equivalence of Artin algebras, Proc. Conf. on Orders, Group Rings and Related Topics, Lecture Notes in Math., vol. 353, Springer-Verlag, Berlin and new York, 1972, pp. 8-71.

[Be] D. J. Benson, Modular representation theory: New trends and methods, Lecture Notes in Math., vol. 1081, Springer-Verlag, Berlin and New York, 1984.

[Bs] C. Bessenrodt, The Auslander-Reiten quiver of a modular group algebra revisited, Math. $\mathrm{Z}$. 206 (1991), 25-34.

[BG] K. Bongartz and P. Gabriel, Covering spaces in representation theory, Invent. Math. 65 (1981/1982), 331-378.

[BR] M. C. R. Butler and C. M. Ringel, Auslander-Reiten sequences with few middle terms and applications to string algebras, Comm. Algebra 15 (1987), 145-179.

[BS] M. C. R. Butler and M. Shahzamanian, The construction of almost split sequences III: modules over two classes of tame local algebras, Math. Ann. 247 (1980), 111-122.

[C] J. Carlson, The dimensions of periodic modules over modular group algebras, Illinois J. Math. 23 (1979), 295-306.

[CB1] W. Crawley-Boevey, On tame algebras and BOCS's, Proc. London Math. Soc. 56 (1988), 451-483.

[CB2] _ Functorial filtrations III: Semidihedral algebras, J. London Math. Soc. 40 (1989), 31-39.

[DS] P. Dowbor and A. Skowroński, Galois coverings of representation-infinite algebras, Comment. Math. Helv. 62 (1987), 311-337.

[D] Ju. A. Drozd, Tame and wild matrix problems, Proc. ICRA II (Ottawa 1979), Lecture Notes in Math., vol. 832, Springer-Verlag, Berlin and New York, 1980, pp. 242-258.

[E1] K. Erdmann, Algebras and dihedral defect groups, Proc. London Math. Soc. 54 (1987), 88-114.

[E2] _ Blocks of tame representation type and related algebras, Lecture Notes in Math., vol. 1428, Springer-Verlag, Berlin and New York, 1990. 
[G] P. Gabriel, The universal cover of a representation-finite algebra, Proc. ICRA III (Puebla 1980), Lecture Notes in Math., vol. 903, Springer-Verlag, Berlin and New York, 1981, pp. 68-105.

[GP] I. M. Gelfand and V. A. Ponomarev, Indecomposable representations of the Lorentz group, Uspekhi Mat. Nauk 23 (1968), 3-60.

[HB] B. Huppert and N. Blackburn, Finite groups. II, Springer-Verlag, Berlin and New York, 1982.

[HPR] D. Happel, U. Preiser and C. M. Ringel, Vinberg's characterization of Dynkin diagrams using subadditive functions with application to DTr-periodic modules, Proc. ICRA II (Ottawa 1979), Lecture Notes in Math., vol. 832, Springer-Verlag, Berlin and New York, 1980, pp. 280-294.

[HW] D. Hughes and J. Waschbüsch, Trivial extensions of tilted algebras, Proc. London Math. Soc. 46 (1983), 347-364.

[K] S. Kawata, Module correspondence in Auslander-Reiten quivers for finite groups, preprint 1988.

[L] P. A. Linnell, The Auslander-Reiten quiver of a finite group, Arch. Math. 45 (1985), 289-295.

[MR] E. Marmolejo and C. M. Ringel, Modules of bounded length in Auslander-Reiten components, Arch. Math. 50 (1988), 128-133.

[O] T. Okuyama, On the Auslander-Reiten quiver of a finite group, J. Algebra 110 (1987), 425430.

[PS] Z. Pogorzały and A. Skowroński, Selfinjective biserial standard algebras, J. Algebra 138 (1991), 491-504.

[Rd] C. Riedtmann, Algebren, Darstellungsköcher, Überlagerungen und Zurück, Comment. Math. Helv. 55 (1980), 199-224.

[R] C. M. Ringel, The representation type of local algebras, Proceedings ICRA I, Lecture Notes in Math., vol. 488, Springer-Verlag, Berlin and New York, 1975.

[R1] - Tame algebras, Proc. Workshop ICRA II (Ottawa 1979), Lecture Notes in Math., vol. 831, Springer-Verlag, Berlin and New York, 1980, pp. 137-287.

[R2] _ Tame algebras and integral quadratic forms, Lecture Notes in Math., vol. 1099, Springer-Verlag, Berlin and New York, 1984.

[S1] A. Skowroński, Group algebras of polynomial growth, Manuscripta Math. 59 (1987), 499516.

[S2] _ Selfinjective algebras of polynomial growth, Math. Ann. 285 (1989), 177-199.

[S3] _ Algebras of polynomial growth, Topics in Algebra, Banach Center Publ., vol. 26, PWN, Warsaw, 1991.

[SW] A. Skowroński and J. Waschbüsch, Representation-finite biserial algebras, J. Reine Angew. Math. 345 (1983), 172-181.

[WW] B. Wald and J. Waschbüsch, Tame biserial algebras, J. Algebra 95 (1985), 480-500.

[W] P. Webb, The Auslander-Reiten quiver of a finite group, Math. Z. 179 (1982), 97-121.

[Z] Zhang Yingbo, The structure of stable components, Doctoral Thesis, Bielefeld, 1990.

Mathematical Institute, University of OXford, 24-29 St. Giles, OXford, England

Institute of Mathematics, Nicholas Copernicus University, Chopina 12/18, 87-100 TORUŃ, POLAND 